\title{
Regional astrocyte IFN signaling restricts pathogenesis during neurotropic viral infection
}

\author{
Brian P. Daniels, ${ }^{1,2}$ Harsha Jujjavarapu, ${ }^{3}$ Douglas M. Durrant,, ${ }^{3,4}$ Jessica L. Williams, ${ }^{3}$ Richard R. Green, ${ }^{2,5}$ James P. White, $^{3}$ \\ Helen M. Lazear, ${ }^{3,6}$ Michael Gale Jr., ${ }^{2,5}$ Michael S. Diamond, ${ }^{3,7,8}$ and Robyn S. Klein ${ }^{1,3,7}$ \\ 'Department of Anatomy and Neurobiology, Washington University School of Medicine, St. Louis, Missouri, USA. Department of Immunology, University of Washington, Seattle, Washington, USA. \\ ${ }^{3}$ Department of Internal Medicine, Washington University School of Medicine, St. Louis, Missouri, USA. ${ }^{4}$ Department of Biological Sciences, California State Polytechnic University, Pomona, California, USA. \\ ${ }^{5}$ Center for Innate Immunity and Immune Disease, University of Washington, Seattle, Washington, USA. ${ }^{6}$ Department of Microbiology and Immunology, University of North Carolina School of Medicine, \\ Chapel Hill, North Carolina, USA. 'Department of Pathology and Immunology, Washington University School of Medicine, St. Louis, Missouri, USA. ${ }^{8}$ Department of Molecular Microbiology, Washington \\ University School of Medicine, St. Louis, Missouri, USA
}

\begin{abstract}
Type I IFNs promote cellular responses to viruses, and IFN receptor (IFNAR) signaling regulates the responses of endothelial cells of the blood-brain barrier (BBB) during neurotropic viral infection. However, the role of astrocytes in innate immune responses of the BBB during viral infection of the CNS remains to be fully elucidated. Here, we have demonstrated that type I IFNAR signaling in astrocytes regulates BBB permeability and protects the cerebellum from infection and immunopathology. Mice with astrocyte-specific loss of IFNAR signaling showed decreased survival after West Nile virus infection. Accelerated mortality was not due to expanded viral tropism or increased replication. Rather, viral entry increased specifically in the hindbrain of IFNAR-deficient mice, suggesting that IFNAR signaling critically regulates BBB permeability in this brain region. Pattern recognition receptors and IFN-stimulated genes had higher basal and IFN-induced expression in human and mouse cerebellar astrocytes than did cerebral cortical astrocytes, suggesting that IFNAR signaling has brain region-specific roles in CNS immune responses. Taken together, our data identify cerebellar astrocytes as key responders to viral infection and highlight the existence of distinct innate immune programs in astrocytes from evolutionarily disparate regions of the CNS.
\end{abstract}

\section{Introduction}

Type I IFNs $(\mathrm{IFN} \alpha / \beta)$ promote the expression of antiviral molecules in response to induction by several different transmembrane and cytosolic pattern recognition receptors (PRRs). Cellular responses to type I IFNs depend on binding to the heterodimeric receptor IFNAR, which initiates a signaling cascade that promotes nuclear translocation of STAT1/2 heterodimers and transcriptional activation of IFN-stimulated genes (ISGs) (1). The rapid expression of hundreds of ISGs is critical for controlling viral infections, as these proteins block viral entry, translation, transcription, assembly, and egress (1-3). However, recent studies indicate that IFNAR signaling also regulates other pathways that impact cellintrinsic antiviral functions. This is particularly evident in studies of IFNAR signaling by cellular constituents of the blood-brain barrier (BBB) during neurotropic viral infection (4). The BBB, which protects the CNS from pathogens, is composed of specialized brain microvascular endothelial cells (BMECs) joined by tight junctions (TJs) and ensheathed by pericytes and astrocyte endfeet

\footnotetext{
Note regarding evaluation of this manuscript: Manuscripts authored by scientists associated with Duke University, The University of North Carolina at Chapel Hill, Duke-NUS, and the Sanford-Burnham Medical Research Institute are handled not by members of the editorial board but rather by the science editors, who consult with selected external editors and reviewers.

Conflict of interest: The authors have declared that no conflict of interest exists.

Submitted: May 20, 2016; Accepted: December 6, 2016.

Reference information: / Clin Invest. 2017;127(3):843-856

https://doi.org/10.1172/JCl88720.
}

(5). While IFNAR signaling in both BMECs and astrocytes contributes to TJ integrity in vitro (4), the in vivo contribution of IFNAR signaling within astrocytes, which exhibit individual and regional heterogeneity, has not been explored.

Innate immune mechanisms that regulate BBB function in the setting of infectious diseases have been appreciated only recently. Multiple inflammatory cytokines, including TNF- $\alpha$, IL-6, IL-1 $\beta$, and IFN- $\gamma$, disrupt BBB and TJ integrity in BMECs through incompletely understood mechanisms (4, 6-8). Inflammatory cytokine signaling at the BBB during infection facilitates leukocyte trafficking into the CNS, which is essential for clearance of many pathogens $(9,10)$. However, this process may have pathological consequences, as the CNS is susceptible to immune cell-mediated injury and possesses a limited capacity for repair (11). Moreover, increased BBB permeability due to inflammatory cytokine signaling also may facilitate viral neuroinvasion (6). Thus, cytokine signaling in BBB constituent cells must be regulated to protect the CNS from infection and inflammation. In contrast to inflammatory cytokines, type I IFNs stabilize BBB integrity and enhance TJ formation via balanced activation of the small GTPases Rac1 and RhoA and by blocking expression of IL-1 $\beta$, which induces barrier disruption (4). Through these actions, type I IFNs serve a protective function at the BBB by counterbalancing the effects of inflammatory cytokines.

Astrocytes have critical roles in host defense during viral infections of the CNS (12). PRR activation in astrocytes results in the expression of many immune mediators, including inflammatory cytokines and type I IFNs $(12,13)$. During infection by patho- 
gens for which glia are not permissive targets, activation due to pathogen recognition and/or abortive infection in astrocytes may also promote antiviral immune responses of permissive neurons as well as CNS leukocyte trafficking $(6,12,14)$. Throughout the CNS, astrocytes exhibit individual and regional heterogeneity in gene expression, morphology, developmental ontogeny, and signaling functions (15). Although in vitro studies have established the fact that astrocytes from different CNS regions respond differently to pathogenic stimuli $(16,17)$, the contributions of astrocyte heterogeneity to in vivo disease pathogenesis and host defense are unknown.

In this study, we investigated how IFNAR signaling in astrocytes impacts the neuropathogenesis of West Nile virus (WNV) encephalitis. Neurons are by far the preferred cellular target of WNV throughout the CNS $(12,18,19)$; in contrast, infection of glial cells in vivo has not been well documented in mice and is probably rare (20). However, astrocyte activation and gliosis has been observed in vivo during WNV infection $(9,21)$. Given the findings from our own studies demonstrating that IFNAR signaling in astrocytes enhances in vitro BBB integrity in response to WNV (4), we used Ifnar ${ }^{l / f l} G f a p$-Cre mice, in which IFNAR signaling was abrogated in glial fibrillary acidic protein-expressing (GFAP-expressing) astrocytes, to evaluate the contribution of astrocyte IFNAR signaling to WNV pathogenesis and disease. For these studies, we chose the Gfap-Cre line 77.6, which was previously shown to exhibit a Cre expression that is more specific to astrocytes than to other GfapCre lines (22-25). We also used an established in vitro BBB model to examine how forebrain versus hindbrain astrocytes differentially regulate barrier function. A deficiency in astrocyte IFNAR signaling in mice with WNV encephalitis resulted in increased BBB permeability specifically within the hindbrain. WNV-infected Ifnart/fl Gfap-Cre ${ }^{+}$mice exhibited decreased survival without increased viral replication or expanded tropism within hindbrain regions, but sustained immunopathologic neuronal death that could be mitigated via functional blockade of the leukocyte adhesion molecule VLA-4. Analysis of expression of PRRs and ISGs revealed higher baseline and IFN-induced levels in human and mouse cerebellar astrocytes compared with levels in cerebral cortical astrocytes. Our findings demonstrate an unrecognized function for astrocyte IFNAR signaling in the regulation of cerebellar BBB permeability and indicate that astrocytes from evolutionarily distinct CNS regions show unique host defense programs.

\section{Results}

WNV infection in mice with global deletion of Ifnar leads to rapid death due to virus-induced sepsis (26). To assess the specific role of IFNAR signaling in astrocytes during WNV infection, we used 8-week-old Ifnar ${ }^{f / f l} \mathrm{Gfap}-\mathrm{Cre}^{+}$and Ifnar ${ }^{f / f l} \mathrm{Cre}^{-}$littermate controls. Analysis of IFNAR expression in uninfected Ifnarl/fl $G f a p-\mathrm{Cre}^{+}$ mice showed low expression of IFNAR in ALDH1L1 ${ }^{+}$astrocytes in both the cerebral cortex (12.5\%) and cerebellum (10.7\%) compared with expression levels in controls (Supplemental Figure 1, $\mathrm{A}$ and $\mathrm{B}$; supplemental material available online with this article; https://doi.org/10.1172/JCI88720DS1). We did not observe substantial deletion of Ifnar in nonastrocytic cells, as IFNAR expression on ALDH1L1 ${ }^{-}$cells remained intact compared with levels detected in controls in both cerebral cortex (95.7\%) and cerebellum (96.1\%). Analysis of IFNAR expression on MAP2 ${ }^{+}$neurons and $\mathrm{IBA}^{+}$microglia also revealed no significant deletion of Ifnar in these cell populations (Supplemental Figure 2, A and B). IFNAR antibody specificity was confirmed by stains using an isotype control antibody as well as tissue derived from Ifnar1 ${ }^{1^{-1}}$ mice (Supplemental Figure 2C). We also observed similar levels of Cre recombinase expression in both cerebral cortex and cerebellum (Supplemental Figure 2D).

Following s.c. inoculation with $10^{2} \mathrm{PFU}$ of WNV (New York 2000 strain), Ifnar ${ }^{f / f l} \mathrm{Gfap}-\mathrm{Cre}^{+}$mice had accelerated and enhanced mortality (16.7\% vs. $50.0 \%$ survival; Figure $1 \mathrm{~A})$ and greater clinical signs of disease (Figure 1B) compared with Ifnar ${ }^{f / f l} \mathrm{Cre}^{-}$controls. In contrast, survival after WNV infection was unaffected in Ifnar ${ }^{f / W T}$ Gfap-Cre ${ }^{+}$littermates or in congenic Gfap-Cre ${ }^{+}$mice that had never been crossed with the Ifnarl/fl line. As expected, viral burden did not differ in any peripheral tissue compartments analyzed, including serum, spleen, kidney, liver, and heart (Figure 1C and Supplemental Figure 3), while viral burden in the CNS was significantly higher in Ifnar ${ }^{f / / f l} \mathrm{Gfap}-\mathrm{Cre}^{+}$mice compared with Ifnar ${ }^{f / / f l} \mathrm{Cre}^{-}$controls. Virus was detected earlier in cerebellar tissues in Ifnar ${ }^{\mathrm{fl} / \mathrm{ll}} \mathrm{Gfap}-\mathrm{Cre}^{+}$mice on day 5 following infection (4 of 7 mice), when levels in Ifnart/fl $\mathrm{Cre}^{-}$controls were undetectable (Figure 1D). By day 6, viral burden in Ifnarl/fl $\mathrm{Gfap}$-Cre $\mathrm{Cr}^{+}$mice remained significantly higher in the cerebellum, while titers in the cerebral cortex and brainstem were elevated but did not reach statistical significance $(P=0.07$ and 0.14 , respectively). By day 8 , viral burden was significantly increased in Ifnar ${ }^{f / f l} \mathrm{Gfap}-\mathrm{Cre}^{+}$mice in all CNS regions analyzed, including the cerebral cortex, brainstem, and spinal cord (Figure 1, D-G).

The early appearance of virus within the cerebellum of Ifnart/fl Gfap-Cre ${ }^{+}$mice suggested that astrocyte IFNAR signaling might limit neuroinvasion at this site. As previous studies have suggested that early WNV neuroinvasion can be caused by defects in BBB function $(4,27,28)$, we determined whether $\mathrm{BBB}$ permeability was altered differentially in the cerebellum and cerebral cortex of Ifnar $^{f / f l} \mathrm{Gfap}-\mathrm{Cre}^{+}$mice compared with BBB permeability in Ifnar ${ }^{f / f l}$ $\mathrm{Cre}^{-}$littermate controls. Analysis of extravasation of sodium fluorescein $(376 \mathrm{Da})$ on various days following s.c. infection showed biphasic kinetics, as observed in our previous studies $(4,21)$, with an enhancement of BBB permeability on day 4 after infection, decreased permeability on days 5 and 6 , and a reopening of the BBB on day 8 (Figure $1 \mathrm{H}$ ). While loss of IFNAR signaling in astrocytes led to enhancement of BBB permeability in both cerebral cortical and cerebellar tissues after infection, the kinetics of these effects differed between regions. In the cerebral cortex, genotype differences reached statistical significance only on days 6 and 8, whereas the ability of the BBB to close on day 5 after infection was attenuated significantly within the cerebellum (Figure $1 \mathrm{H}$ ). Using immunohistochemical detection of extravasated endogenous IgG $(150 \mathrm{kDa})$, we observed enhanced $\mathrm{BBB}$ permeability to larger molecules on day 6 in the cerebellum but not the cerebral cortex (Figure 1, I and J). The defect in BBB function on days 5 and 6 following infection coincided with the early detection of virus in the cerebellum, suggesting that astrocyte IFNAR signaling in the cerebellum might be critical for regulating $\mathrm{BBB}$ responses that limit viral neuroinvasion.

Apart from its regulation of BBB function, IFN signaling in astrocytes may also serve protective antiviral functions for neurons $(14,29)$, which might contribute to higher CNS titers at early time 

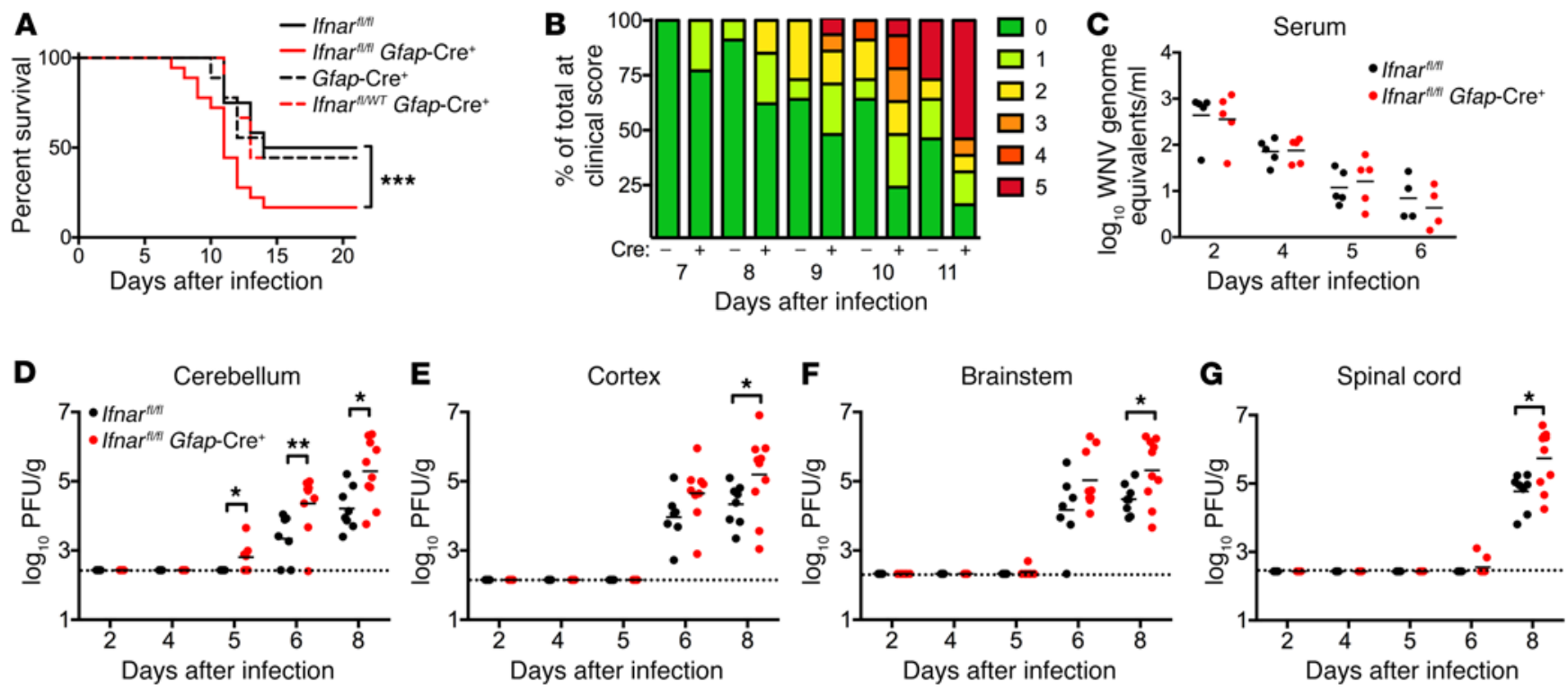

H

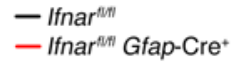
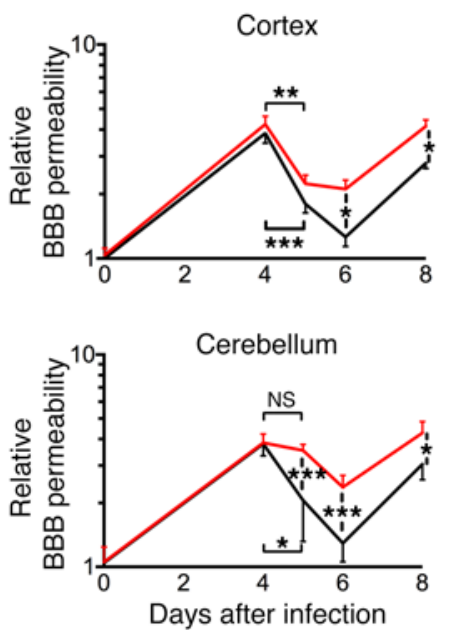

I
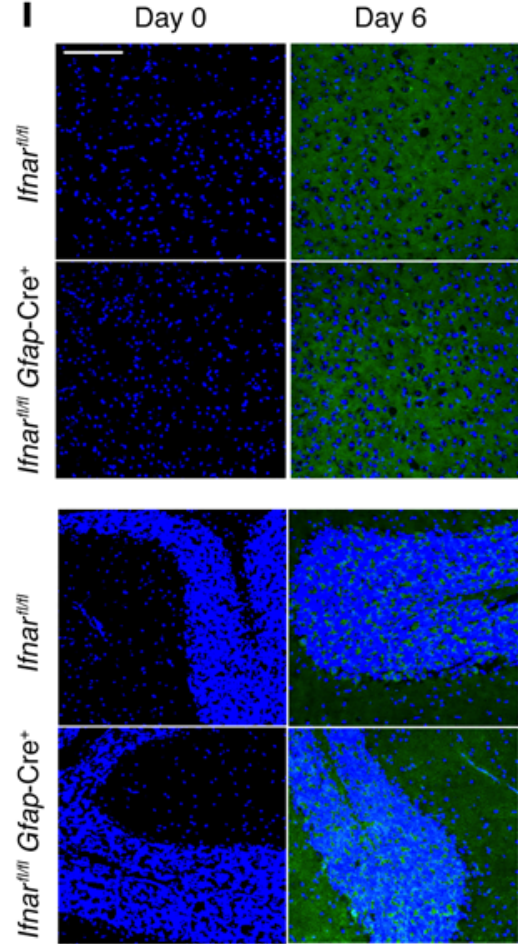

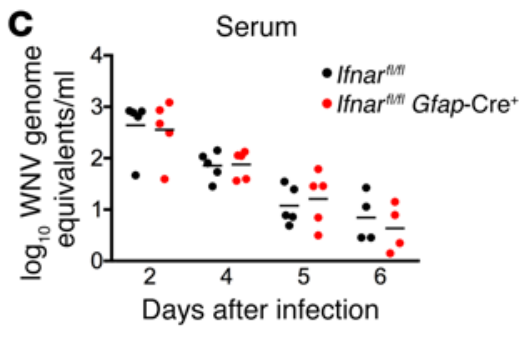

$\mathbf{J}$
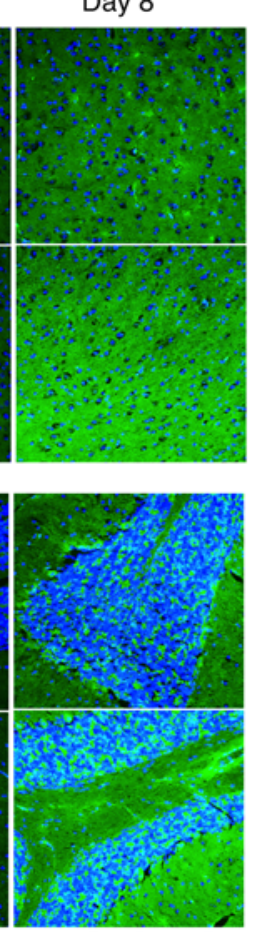

$\operatorname{lgG}$ TO-PRO-3
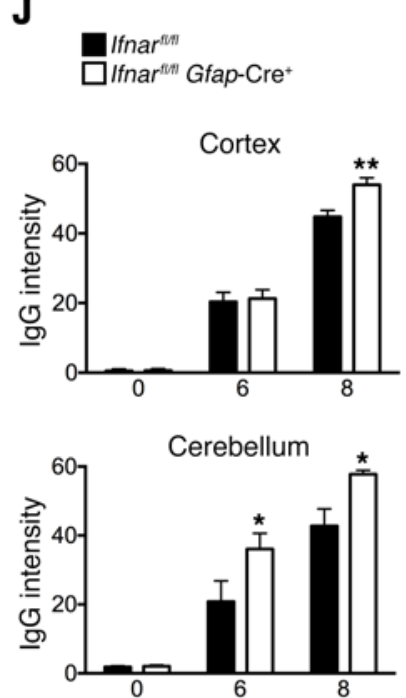

Days after infection

Figure 1. Survival and viral burden following s.c. WNV inoculation. (A-I) Mice were inoculated s.c. with WNV (New York 2000 strain). (A) Mice were monitored daily for survival after infection. (B) Clinical scores of Ifnar ${ }^{f / f l}$ mice either positive or negative for Gfap-Cre ( $x$ axis) were recorded on the indicated post-infection days. 0 = subclinical; 1 = hunched/ruffled fur; $2=$ altered gate/slow movement; $3=$ no movement, but responsive to stimuli; $4=$ moribund; 5 = dead. (C) Serum viral loads as measured by qRT-PCR. (D-C) Tissue viral loads as measured by plaque assay. (H) BBB permeability was measured by detection of sodium fluorescein accumulation in tissue homogenates derived from cerebral cortex or cerebellum. Data represent the mean \pm SEM of individual mouse values normalized to serum sodium fluorescein concentration. Group means were then normalized to the mean values for uninfected controls. (I and J) Immunohistochemical detection of endogenous IgG accumulation in parenchymal CNS tissues. Signal intensities were quantified from two $\times 40$ fields per region using Imagej software. Scale bar: $100 \mu \mathrm{m}$. Data in $\mathbf{A}$ and $\mathbf{B}$ represent pooled data collected from 3 independent experiments for Ifnar ${ }^{f l / f l} \mathrm{Cre}^{-}(n=12)$, Ifnar ${ }^{f l / f l} \mathrm{Cfap}-\mathrm{Cre}^{+}(n=17)$, or Ifnar ${ }^{-1-}(n=8)$ mice. Data in C-G were collected from 2 to 3 independent experiments and represent values recorded for 4 to 10 mice per time point. Data in G-I were pooled from a total of 5 mice per time point and were collected from 2 independent experiments. ${ }^{*} P<0.05,{ }^{* *} P<0.01$, and ${ }^{* *} P<0.001$, by log-rank test $(\mathbf{A})$, Mann-Whitney $U$ test $(\mathbf{C}-\mathbf{C})$, or 2 -way ANOVA (H and $\left.\mathbf{J}\right)$. 
A
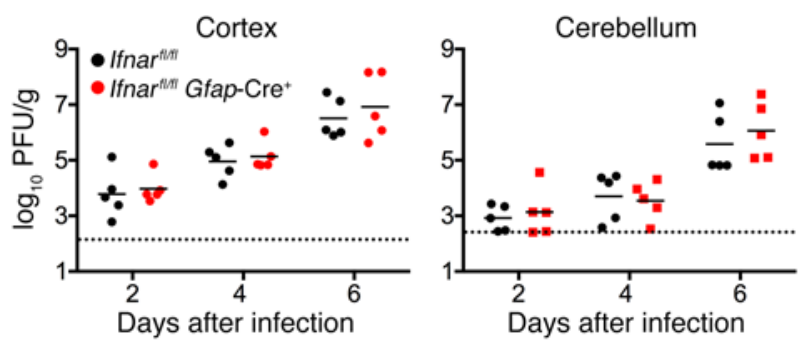

C

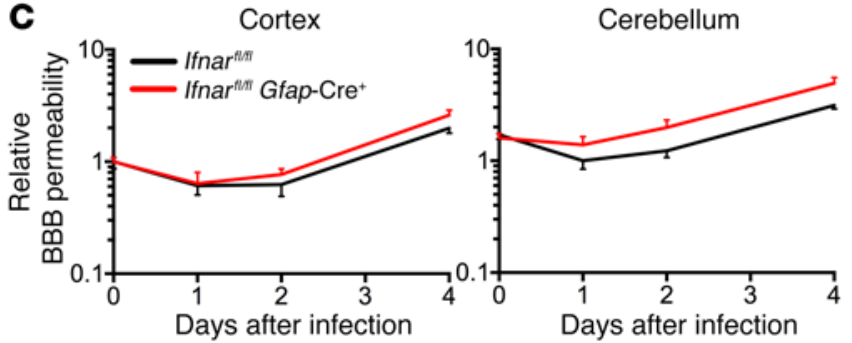

B
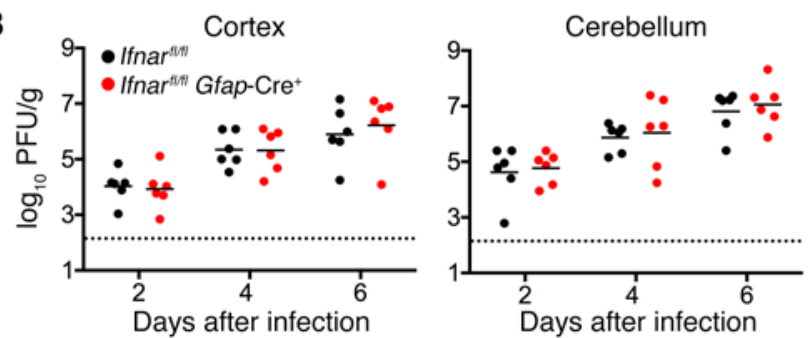

D
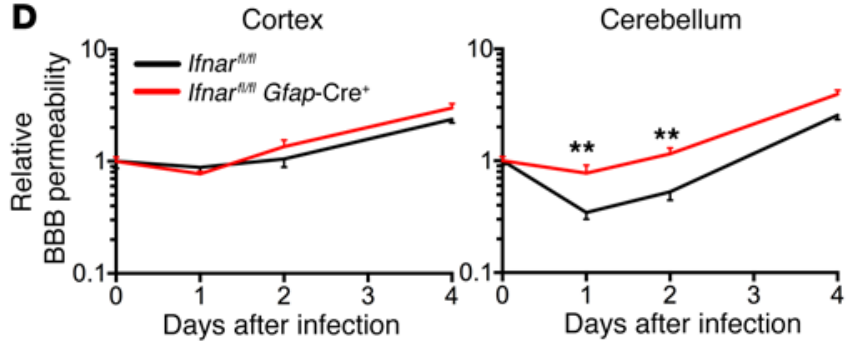

Figure 2. CNS replication and BBB permeability following intracranial inoculation. (A-D) Mice were inoculated with WNV (New York 2000 strain) via the intracranial route, and CNS tissues were harvested on the indicated post-infection days. (A and B) Cerebral cortex and cerebellar tissues were collected following intracranial inoculation within the cortex (A, intracortical inoculation) or cerebellum (B, intracerebellar inoculation). WNV titers were determined by standard plaque assay. (C and D) Cerebral cortex and cerebellar tissues were analyzed for accumulation of sodium fluorescein in tissue parenchyma following intracranial inoculation within the cortex (C, intracortical inoculation) or cerebellum (D, intracerebellar inoculation). Data represent the mean \pm SEM of individual mouse values normalized to serum fluorescein concentration. Group means were then normalized to mean values for uninfected controls. Data in A-D were collected from 2 independent experiments and represent the mean values taken from a total of 5 mice per time point. Data were analyzed by Mann-Whitney $U$ test (A and $\mathbf{B}$ ) or 2-way ANOVA (C and D). ${ }^{*} P<0.01$.

points independently of enhanced neuroinvasion due to increased BBB permeability. To differentiate these hypotheses, we infected mice with WNV via an intracranial route, allowing us to bypass barriers to neuroinvasion and measure the kinetics of viral replication in CNS neurons directly. Viral titers did not differ between genotypes in cerebral cortical or cerebellar tissues following intracranial inoculation with $10 \mathrm{PFU}$ of WNV, regardless of whether virus was inoculated directly into the cerebral cortex (Figure 2A) or cerebellum (Figure 2B). Similar results were obtained following intracerebellar inoculation with 10 PFU of WNV-Madagascar (Supplemental Figure 4), an attenuated strain that is more IFN sensitive than are highly pathogenic strains of WNV (30). Analogous to tissue titers, BBB permeability did not differ significantly between genotypes following intracortical inoculation with WNV (Figure 2C), although cerebellar tissues showed a trend toward increased permeability. However, following intracerebellar inoculation, BBB closure was diminished significantly in the cerebellum at early time points following infection of Ifnar ${ }^{f / f l} \mathrm{Gfap}-\mathrm{Cre}^{+}$mice compared with Ifnar ${ }^{f / / f l} \mathrm{Cre}^{-}$littermate controls (Figure 2D), suggesting that type I IFN signaling in astrocytes sustains BBB integrity following detection of WNV by cerebellar tissues. Importantly, we observed no expansion of viral tropism to non-neuronal cells in Ifnar ${ }^{f / f l} \mathrm{Gfap}-\mathrm{Cre}^{+}$mice following either s.c. (data not shown) or intracerebellar inoculation (Supplemental Figure 5), suggesting that disease burden in these mice is not driven by an aberrant susceptibility of astrocytes and other glia to infection. Together, these results suggest that higher viral titers in the cerebellum following s.c. infection in Ifnarl/fl $G$ fap-Cre ${ }^{+}$mice are due to defects in BBB restriction of neuroinvasion in the cerebellum during the early stages of CNS infection.
We questioned whether enhanced BBB permeability and WNV neuroinvasion in the cerebellum of Ifnar ${ }^{f / f l} \mathrm{Gfap}-\mathrm{Cre}^{+}$mice were due to alterations in the regional expression of vasoactive cytokines, including type I IFNs, TNF- $\alpha$, and IL- $1 \beta$, as our previous studies showed that cytokine-mediated regulation of BBB permeability can directly influence WNV movement across endothelial barriers $(4,27,28)$. On day 6 after s.c. inoculation with WNV, control Ifnar ${ }^{f l / f l} \mathrm{Cre}^{-}$cerebellar tissues showed higher levels of Ifnb (Figure 3A) and Ifna (Figure 3B) mRNA compared with cerebral cortex tissues with lower expression of the proinflammatory cytokine Illb (Figure 3C). In contrast, Ifnar ${ }^{f / f l} \mathrm{Gfap}-\mathrm{Cre}^{+}$cerebellar tissues had higher levels of Illb expression than did Ifnar ${ }^{f / f l}$ $\mathrm{Cre}^{-}$littermate controls, whereas expression of this cytokine in the cerebral cortex was unaffected by deletion of astrocytic Ifnar (Figure 3C). We observed no differences in Tnfa expression in either genotype at this time point (Figure 3D). By day 8 after infection, mRNA levels of type I IFNs, Il1b, and Tnfa were higher in tissues from both the cerebral cortex and cerebellum of Ifnar ${ }^{f / f l} \mathrm{Gfap}-\mathrm{Cre}^{+}$ mice, which likely reflected the higher viral titers found across the CNS at this time point (Figure 1, D-G). Differential cytokine gene expression in these tissues was confirmed at the protein level by ELISA (Supplemental Figure 6). These data suggest that enhanced cerebellar BBB permeability and WNV neuroinvasion in Ifnarl/fl Gfap-Cre ${ }^{+}$mice on day 6 (Figure 3, A and B) following s.c. infection are due in part to changes in the relative expression of barrierstabilizing (e.g., type I IFNs) versus barrier-disrupting (e.g., IL-1 $\beta$ ) cytokines (Figure 3, A and B versus Figure 3, C and D) (4).

During the course of these studies, we observed that expression of the inflammatory chemokines CCL2 and CXCL10, which are produced by WNV-infected neurons (31), was higher in cerebel- 


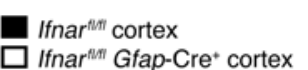

$\square$ Ifnar ${ }^{\text {thI }}$ cerebellum

Ifnar ${ }^{\text {thI }}$ Gfap-Cre ${ }^{+}$cerebellum
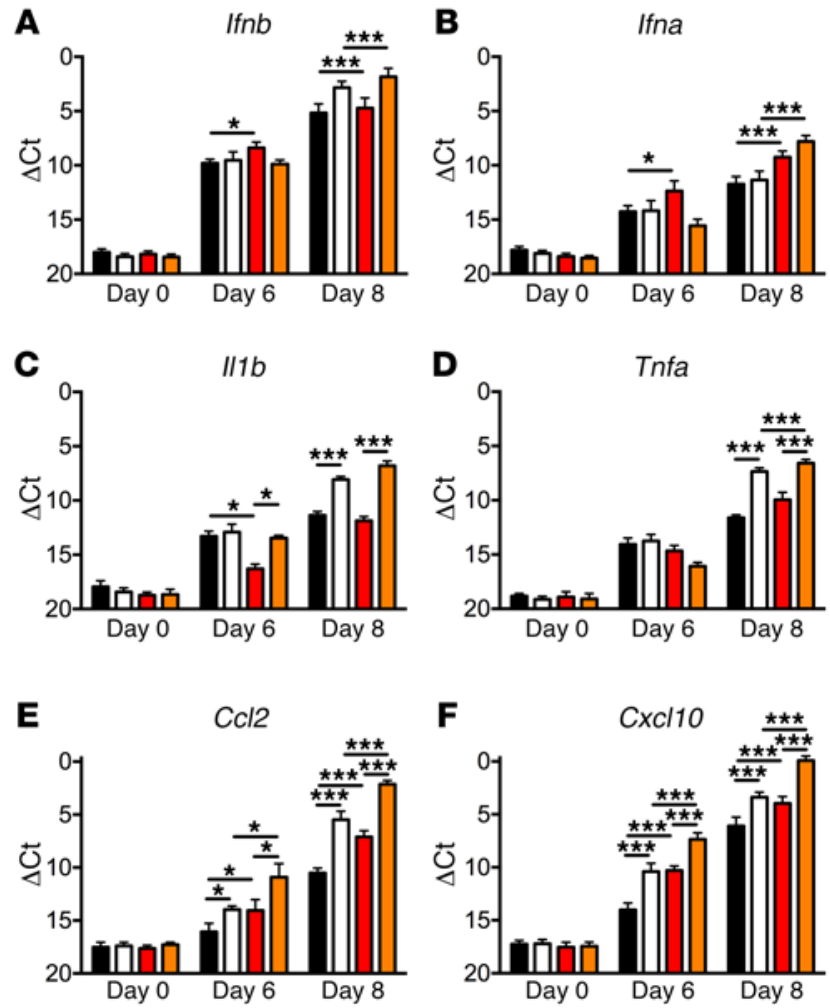

lar tissues following infection, with Ifnar ${ }^{f / f l} \mathrm{Gfap}-\mathrm{Cre}^{+}$mice showing even higher levels of $\mathrm{Ccl} 2$ and $\mathrm{Cxcl} 10$ expression in cerebellar tissues (Figure 3, E and F) than were detected in their littermate Ifnar ${ }^{l / f l} \mathrm{Cre}^{-}$controls. Because of this result, we evaluated whether the increased expression of leukocyte chemoattractant molecules in Ifnarl/fl $G$ fap-Cre ${ }^{+}$mice resulted in differences in CNS immune cell infiltration. Although the total number of infiltrating immune cells in the cerebral cortex was unchanged in Ifnar ${ }^{f / f l}$ Gfap-Cre ${ }^{+}$ mice compared with the total number in Ifnarf/fl $\mathrm{Cre}^{-}$controls on day 8 following s.c. inoculation with WNV (Supplemental Figure $7 \mathrm{~A})$, there were greater numbers of $\mathrm{CD}^{+} \mathrm{IFN}-\gamma^{+}$lymphocytes and $\mathrm{CD} 45^{\mathrm{hi}} \mathrm{CD} 11 \mathrm{~b}^{\mathrm{lo}} \mathrm{F} 4 / 80^{+}$macrophages in the cerebellum (Supplemental Figure 7B). As enhanced inflammatory infiltrates may be a secondary consequence of higher viral titers in Ifnar ${ }^{\text {flflf }} \mathrm{Gfap}$-Cre ${ }^{+}$ mice following s.c. infection, we performed a similar analysis of CNS immune infiltrates on day 6 following intracerebellar inoculation with WNV, as viral titers are indistinguishable between genotypes in this model. These experiments showed no significant differences in immune cell infiltration between genotypes in the cerebral cortex, but again revealed greater infiltration of several leukocyte subsets (e.g., T lymphocytes and macrophages) in the cerebellum of Ifnarl/fl $\mathrm{Gfap}-\mathrm{Cre}^{+}$mice compared with Ifnar ${ }^{f / f l} \mathrm{Cre}^{-}$ littermate controls (Figure 4, A and B). These data suggest that, even in the presence of similar viral titers, type I IFN signaling in cerebellar astrocytes suppresses neuroinflammation.

We questioned whether the increased immune infiltration in the cerebellum of Ifnarl/fl $G$ fap-Cre ${ }^{+}$mice was a pathologic rather than protective response in the setting of astrocyte-specific
Figure 3. Cytokine and chemokine expression in the CNS following s.c. inoculation. (A-F) Mice were s.c. inoculated with WNV. Cerebral cortex and cerebellar tissues were extracted on the indicated post-infection days. Relative transcript levels in tissue homogenates were measured by SYBR qRT-PCR for the indicated cytokines and chemokines. Data for individual mice were normalized to Gapdh. Data represent the mean \pm SEM from a total of 5 to 6 mice and were collected from 2 independent experiments. All data were analyzed by 2 -way ANOVA. ${ }^{*} P<0.05$ and ${ }^{* *} P<0.001$

deficiency of IFNAR signaling. To assess this, we examined survival following intracranial inoculation with WNV. Although all mice succumbed to infection in this model, intracranial infections allowed examination of the potential pathological impact of enhanced immune infiltration in the absence of differential viral titers. Remarkably, while intracortical inoculation with WNV resulted in no significant difference in overall survival rates between genotypes, Ifnar ${ }^{f / f l} \mathrm{Gfap}-\mathrm{Cre}^{+}$mice had accelerated mortality compared with Ifnarf/fl $\mathrm{Cre}^{-}$controls following intracerebellar inoculation (Figure $4 \mathrm{C}$ ). Immunohistochemical analysis revealed a broad parenchymal distribution of $\mathrm{CD}^{+}$lymphocytes (Figure 4D) and infiltrating IBA1 $1^{+} \mathrm{CD} 45^{\text {hi }}$ myeloid cells (Supplemental Figure 7C) in the cerebella of infected Ifnarl/fl $\mathrm{Gfap}-\mathrm{Cre}^{+}$ mice on day 6 following intracerebellar inoculation. Infiltrating $\mathrm{CD}^{+} \mathrm{T}$ cells were associated with $\mathrm{TUNEL}^{+}$apoptotic cells, especially in Ifnarl/fl $\mathrm{Gfap}$-Cre $\mathrm{Cr}^{+}$mice, which had higher numbers of apoptotic neurons and astrocytes compared with numbers detected in Ifnar ${ }^{f l f l} \mathrm{Cre}^{-}$controls (Figure 4, E and F).

As type I IFN regulates immune trafficking by modulating VCAM-1 (CD106) expression on neurovascular cells (32-34), we determined whether the enhanced neuroinflammation in Ifnarl/fl Gfap-Cre ${ }^{+}$mice was associated with increased VCAM-1 expression. While VCAM-1 expression increased across the CNS following WNV infection, Vcam-1 mRNA levels were further increased in the cerebellum but not in the cerebral cortex of Ifnar ${ }^{f / f l}$ Gfap$\mathrm{Cre}^{+}$mice compared with Ifnar ${ }^{f / f l} \mathrm{Cre}^{-}$controls on days 6 and 8 after s.c. inoculation (Figure 5A and Supplemental Figure 8A). IFN- $\beta$ treatment of either human or mouse primary astrocyte cultures from the cerebellum, but not from the cerebral cortex, significantly downregulated VCAM-1 expression (Figure 5B and Supplemental Figure 8B). Immunohistochemical analysis following intracerebellar inoculation of Ifnar ${ }^{f / f l} \mathrm{Gfap}-\mathrm{Cre}^{+}$mice revealed no genotype-dependent differences in VCAM-1 expression in the cerebral cortex (Figure 5C). In contrast, Ifnarl/fl $\mathrm{Gfap}^{\mathrm{f}} \mathrm{Cre}^{+}$cerebellar tissues showed enhanced VCAM-1 expression that was distributed across multiple cell types, including endothelial cells and astrocytes (Figure 5D). While VCAM-1 expression on vascular endothelium is a well-known regulator of neuroinflammation (35), VCAM-1 expression on astrocytes has also been shown to promote neuroinflammation by enhancing the penetration of infiltrating leukocytes out of perivascular spaces and into the CNS parenchyma $(36,37)$. Moreover, pharmacological blockade of VCAM-1 binding to its ligand VLA- 4 has previously been shown to decrease pathogenic neuroinflammation and improve survival following s.c. WNV infection (38). Given that IFNAR signaling in astrocytes downregulated VCAM-1 expression in the cerebellum following WNV infection, we questioned whether VCAM-1 expression contributed to the enhanced immune infiltration and immunopathol- 


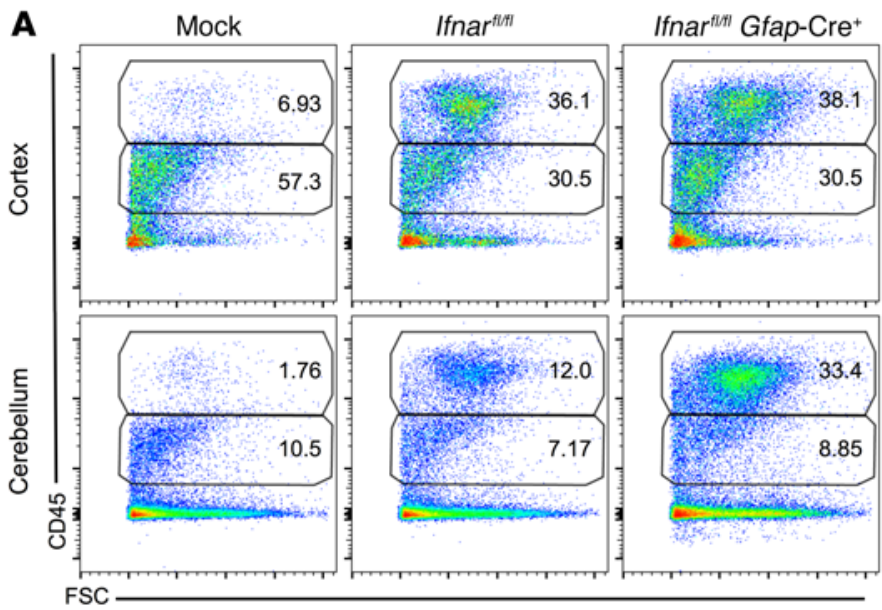

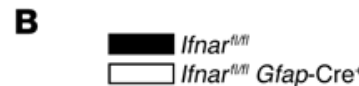

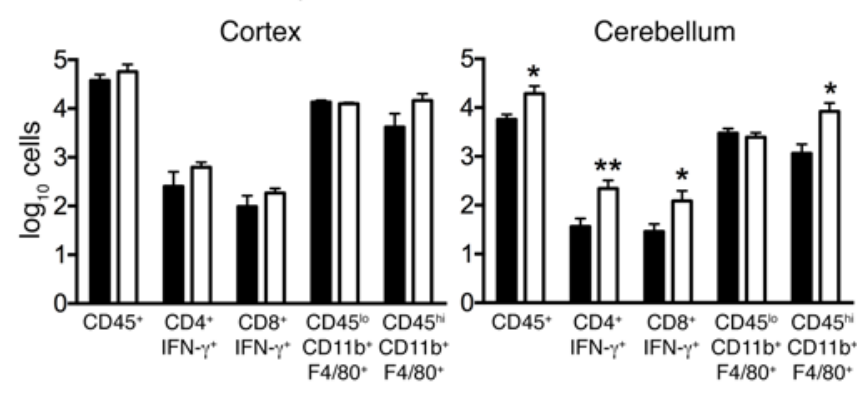

D

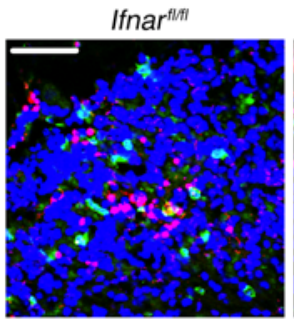

Ifnar ${ }^{f / / I}$ Gfap-Cre ${ }^{+}$

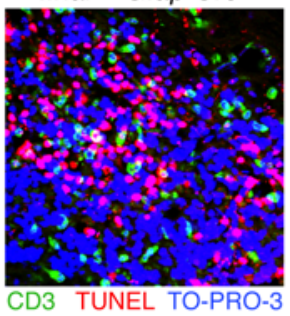

$\mathbf{E}$
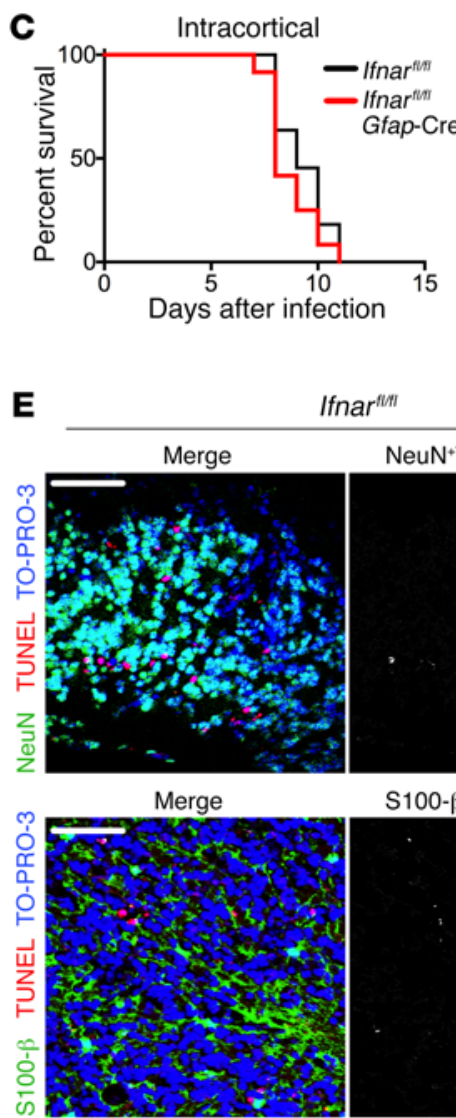

Ifnar ${ }^{\text {IUI }}$

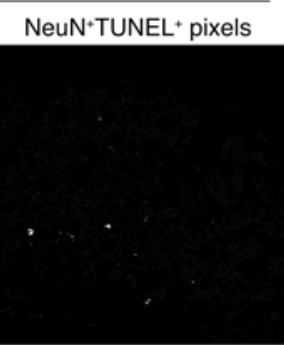

S100- $\beta^{+}$TUNEL ${ }^{+}$pixels

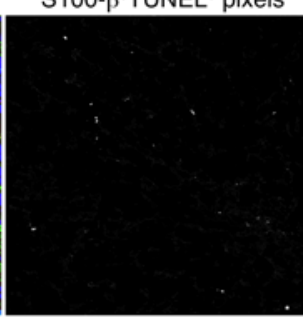

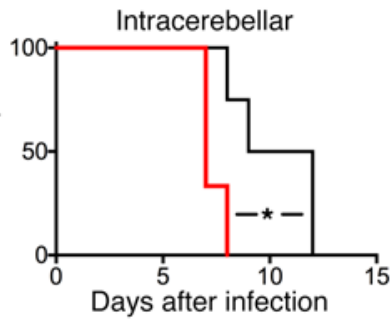

F
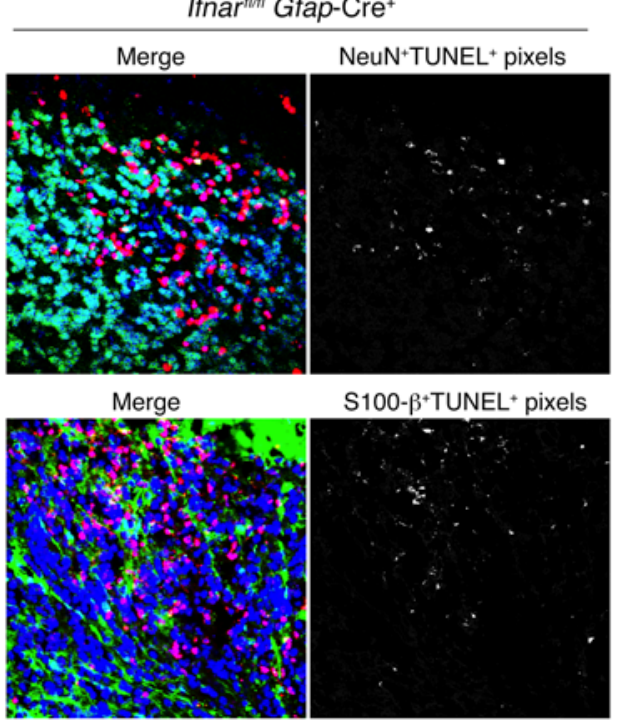

S100- $\beta^{+}$TUNEL + pixels

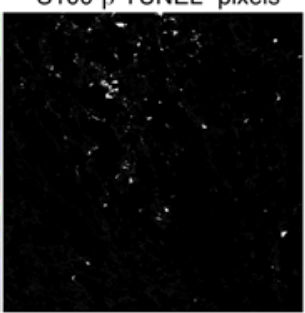

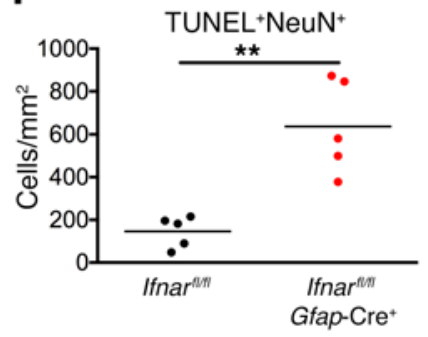

TUNEL+S100- $\beta^{+}$

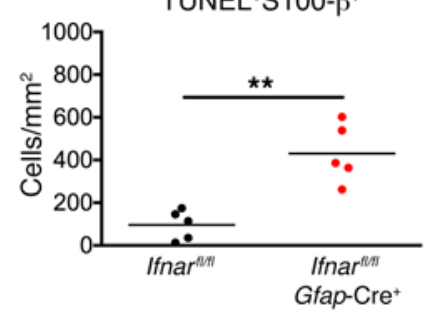

Figure 4. CNS immune cell trafficking and immunopathology following intracranial inoculation. (A and B) Flow cytometric analysis of CNS immune cell infiltrates on day 6 following intracerebellar infection. (A) Representative flow cytometric dot plots demonstrating the percentages of CD45 $5^{\text {hi }}$ versus $C D 45^{\text {lo }}$ brain leukocytes isolated from the cerebral cortex and cerebellum of mock- and WNV-infected mice. The representative panel for mock infection is for an Ifnar ${ }^{f l / f l}$ mouse, which resembles mock infection in Gfap-Cre+ mice (shown here). The numbers indicate the percentage of each cell type within the total number of cells isolated (after exclusion of doublets and dead cell debris). (B) Total number of CD45 leukocytes, CD45 $5^{\text {hi }}$ CD4 $4^{+}$and CD45 $5^{\text {hi }}$ CD8 ${ }^{+}$lymphocytes expressing IFN- $\gamma$, and $C D 45^{\circ} \mathrm{CD} 11 \mathrm{~b}^{+} \mathrm{F} 4 / 80^{+}$microglia and $\mathrm{CD} 45^{\mathrm{h}} \mathrm{CD} 11 \mathrm{~b}^{+} \mathrm{F} 4 / 80^{+}$macrophages isolated from the indicated brain region. Data in $\mathbf{B}$ were collected from 2 independent experiments and represent the mean \pm SEM for 6 mice. Note that data in $\mathbf{A}$ and $\mathbf{B}$ were not normalized for the greater tissue volume of the cerebral cortex versus the cerebellum. (C) Survival was monitored following either intracortical or intracerebellar inoculation. Survival data for 10 to 11 mice per group were pooled from 2 independent infections. ( $\mathbf{D}$ and $\mathbf{E}$ ) Immunohistochemical detection of TUNEL ${ }^{+}$nuclei of dead and dying cells in the cerebellar granule cell layer, costained for $\mathrm{CD3}^{+}$T lymphocytes (D), NeuN ${ }^{+}$neurons (E), or S100- $\beta^{+}$astrocytes (E). Scale bars: $50 \mu \mathrm{m}$. (F) Quantification of dead and dying neurons (TUNEL+NeuN ${ }^{+}$) or astrocytes $\left(T U N E L^{+} S 100-\beta^{+}\right)$, generated by counting cells that were double positive for TUNEL and the appropriate cell marker (depicted in pixel-masked images to the right of the merged RGB images in E). Data were determined by counting 4 images from 2 nonserial sections per mouse ( $n=5$ mice/group). Counts for each image were normalized to the total area of the granule cell layer present per high-power field. Graphed data points represent averages for all images taken for individual mice. All data represent pooled values from 2 independent experiments and were compared using an unpaired, 2 -tailed Student's $t$ test $\left(\mathbf{B}\right.$ and $\mathbf{F}$ ) or log-rank test $(\mathbf{C}) .{ }^{*} P<0.05$ and ${ }^{* *} P<0.01$. All error bars are SEM. 

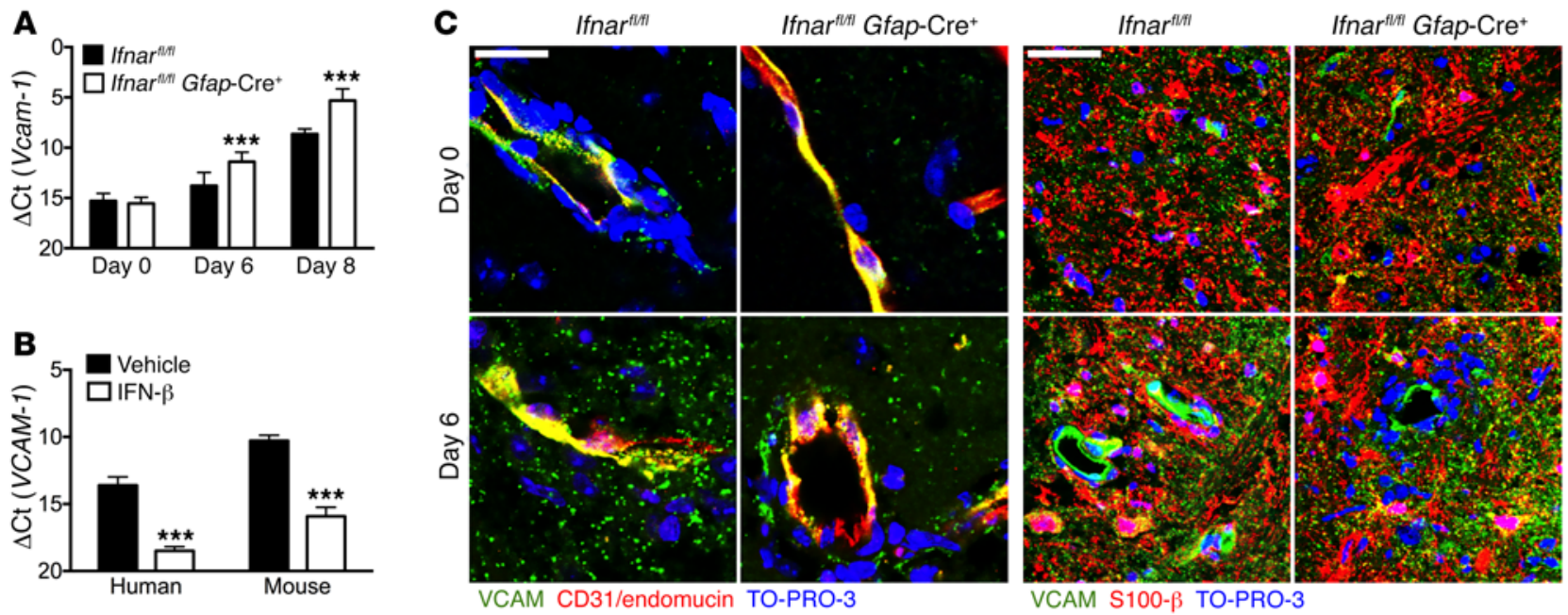

Figure 5. VCAM-1 expression in cerebellar astrocytes in vitro and in the CNS in vivo following infection. (A and B) Vcam-1 mRNA levels were detected by SYBR qRT-PCR. (A) Vcam-1 mRNA levels were detected in the cerebella of mice taken on the indicated days following s.c. infection. (B) VCAM-1 mRNA levels in primary adult human cerebellar astrocytes and primary murine neonatal cerebellar astrocytes following a 4-hour treatment with $10 \mathrm{U} / \mathrm{ml}$ IFN- $\beta$. Ct values in $\mathbf{A}$ and $\mathbf{B}$ were normalized to the $\mathrm{Ct}$ values of the housekeeping gene Gapdh. (C and D) Immunohistochemical detection of VCAM-1 expression on CD31/endomucin ${ }^{+}$(cocktail ${ }^{+}$) endothelial cells and $5100-\beta^{+}$astrocytes in the cerebral cortex (C) or cerebellum (D). Scale bars: $50 \mu \mathrm{m}$. VCAM-1 expression on the indicated cell type was quantified as the mean VCAM-1 (green) fluorescence intensity of pixels that stained positive for the indicated cell marker. Data were obtained after quantifying 4 images from 2 randomly chosen, nonserial sections per mouse $(81,000$ $\mu \mathrm{m}^{2}$ total analyzed area per mouse, 5 mice per group). Data are reported as $\log _{10}$ arbitrary fluorescence intensity units. Data in $\mathbf{A}-\mathbf{D}$ are from 2 independent experiments and are representative images or the mean \pm SEM of 5 to 6 replicates per mouse per group. ${ }^{* * *} P<0.001$, by 2 -way ANOVA.

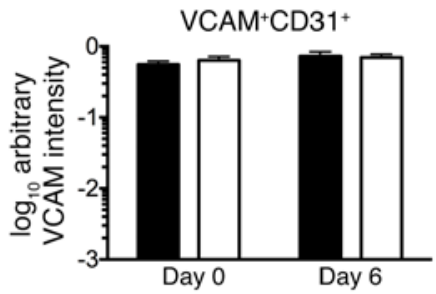

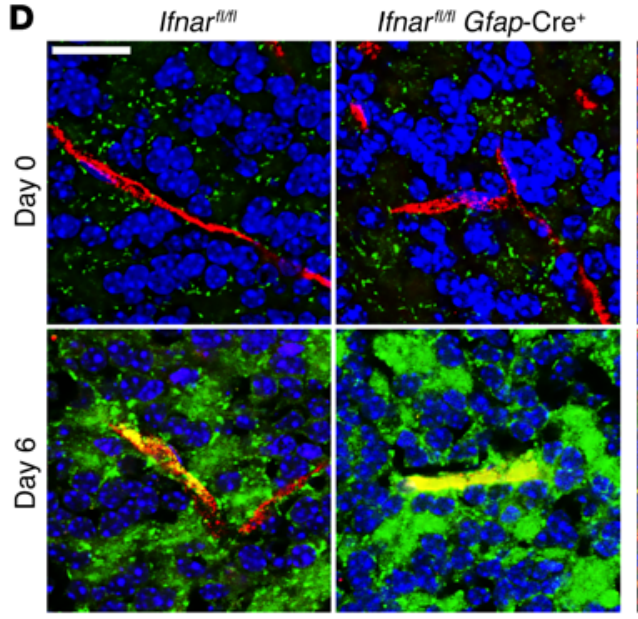

VCAM CD31/endomucin TO-PRO-3

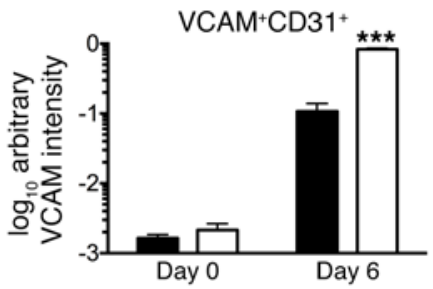

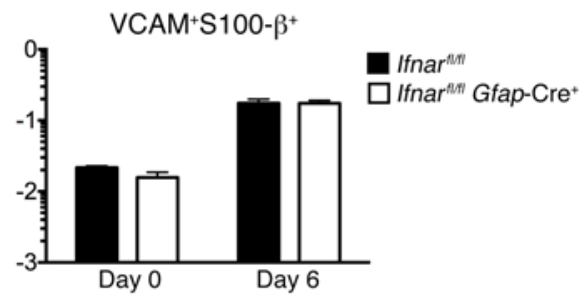

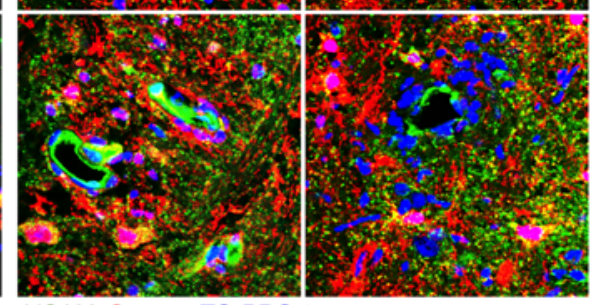

VCAM S100- $\beta$ TO-PRO-3
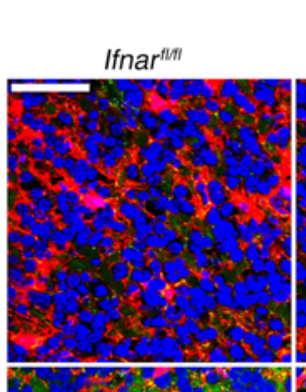

Ifnar ${ }^{\text {fll/I }}$ Gfap-Cre ${ }^{+}$

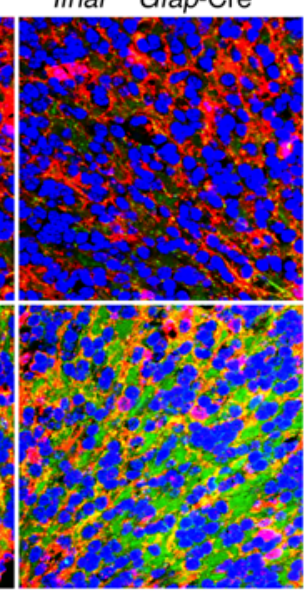

VCAM S100- $\beta$ TO-PRO-3

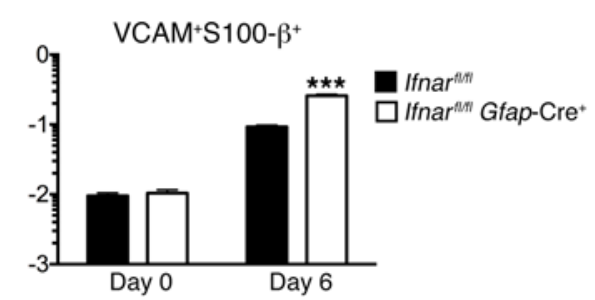

ogy observed in Ifnar ${ }^{f / f l} G f a p-\mathrm{Cre}^{+}$mice. Thus, we tested whether blockade of VCAM-1-VLA-4 binding would protect Ifnar ${ }^{f / f l}$ Gfap$\mathrm{Cre}^{+}$mice following WNV infection. Daily administration of a small-molecule antagonist of VLA-4 (BIO5192) starting on day 4 following intracerebellar infection extended survival in Ifnart/fl Gfap-Cre ${ }^{+}$mice, without impacting survival in the Ifnar ${ }^{f / f l} \mathrm{Cre}^{-}$littermate controls (Figure 6A). Flow cytometric analysis on day 6 after infection revealed that BIO5192 treatment modestly reduced the number of infiltrating lymphocytes and macrophages in Ifnart/fl $\mathrm{Cre}^{-}$littermates, with only differences in macrophage numbers reaching statistical significance (Figure 6B). In contrast, VLA-4 blockade significantly reduced infiltrating lymphocyte and macrophage numbers in Ifnar ${ }^{f / f l} \mathrm{Gfap}-\mathrm{Cre}^{+}$cerebella to levels indistinguishable from those detected in littermate controls. BIO5192 treatment also reduced the numbers of $\mathrm{TUNEL}^{+}$neurons and astrocytes (Figure 6, C and D) in the cerebella of Ifnar ${ }^{f / f l}$ Gfap-Cre ${ }^{+}$ 

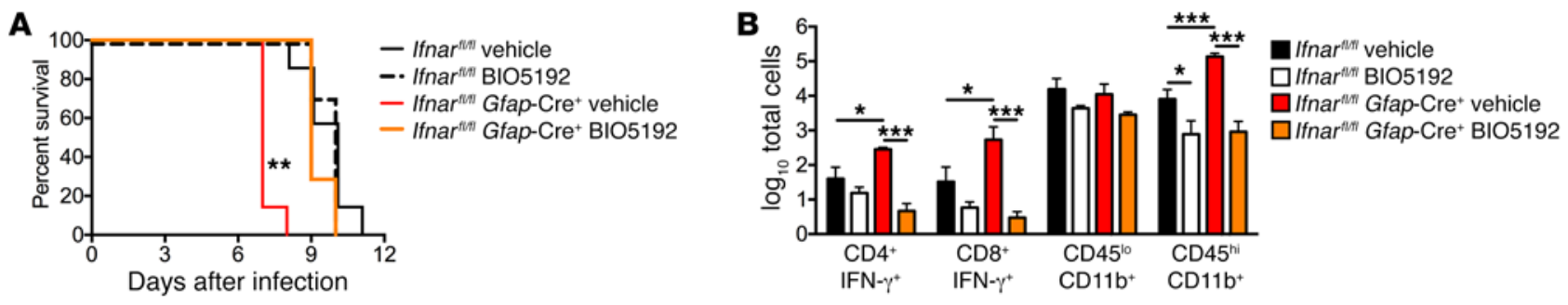

C
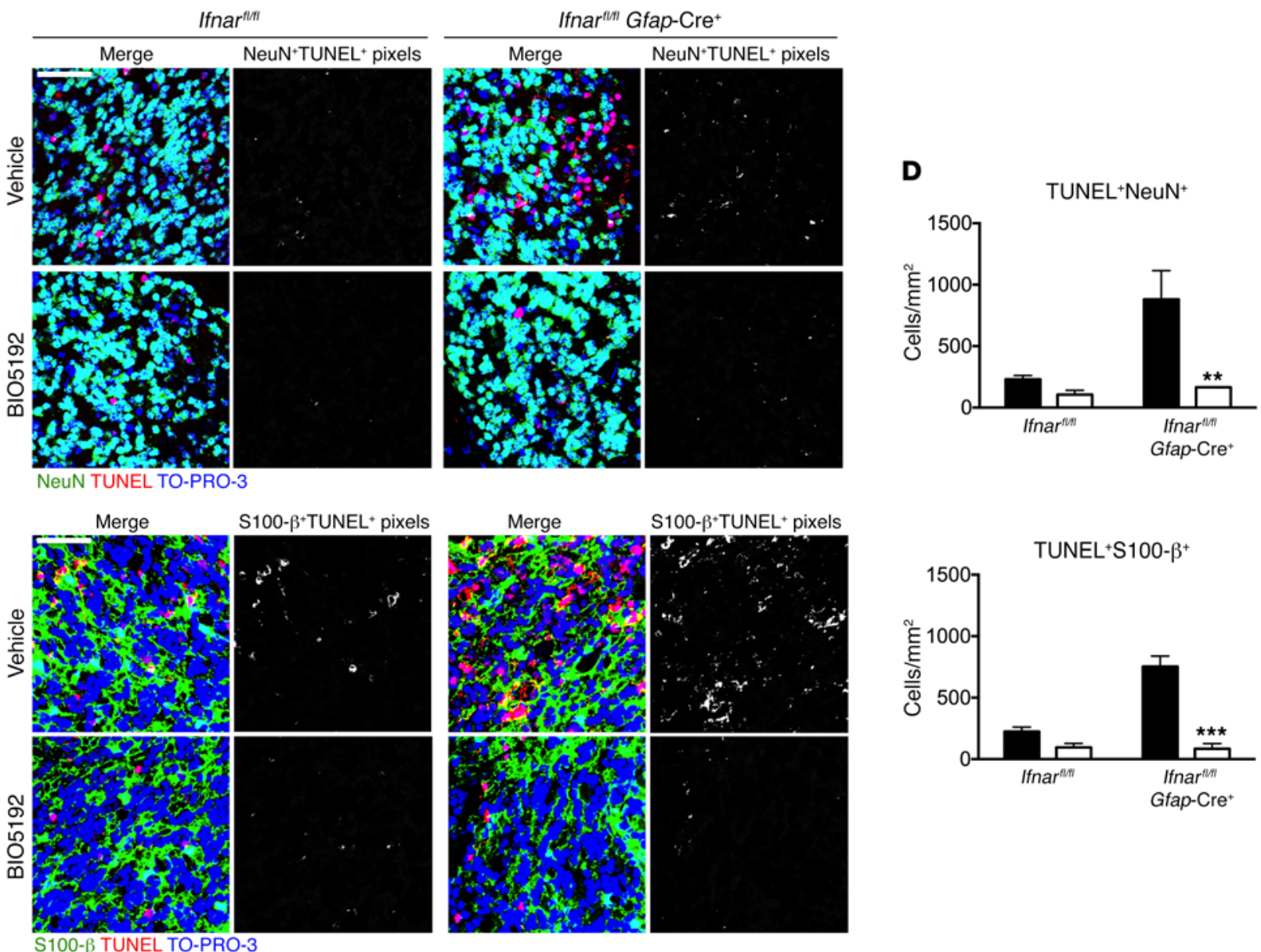

Figure 6. Survival, immune infiltrates, and immunopathology following pharmacological blockade of VLA-4. (A-D) Mice were inoculated via an intracerebellar route with WNV. Infected mice in all experiments were treated i.p. with $10 \mathrm{mg} / \mathrm{kg}$ BIO5192, a small-molecule VLA-4 antagonist, or vehicle solution beginning on day 4 after infection. (A) Survival was monitored daily following infection ( $n=7$ mice/group). (B) Flow cytometric analysis of immune infiltrates in the cerebellum on day 6 after infection ( $n=4$ mice/group) (C) Immunohistochemical detection of TUNEL ${ }^{+}$neurons (NeuN, left) and astrocytes (S100- $\beta$, right) in cerebellar granule cell layers of infected mice on day 6 after infection. Scale bar: $50 \mu \mathrm{m}$. (D) Quantification of dead and dying neurons (left panel) or astrocytes (right panel), generated by counting cells double positive for TUNEL and the appropriate cell marker (depicted in pixel masked images to the right of merged RGB images). Data are taken from counting 4 images from 2 nonserial sections per mouse (5 mice/group). Counts for each image were normalized to the total area of granule cell layer present per high-power field. All data represent pooled values from 2 independent experiments. ${ }^{*} P<0.05,{ }^{* *} P<0.01$, and ${ }^{* *} P<0.001$, by log-rank test $(\mathbf{A})$ or 2-way ANOVA (B and $\mathbf{D}$ ).

mice on day 6 after infection. Thus, blockade of VCAM-1 in Ifnar ${ }^{\text {Ifl }}$ Gfap-Cre ${ }^{+}$mice following WNV infection decreased inflammation and immunopathology specifically in the cerebellum. These data indicate that enhanced expression of leukocyte migratory cues and the resultant neuroinflammation contribute directly to the enhanced CNS tissue injury and animal mortality observed in Ifnar ${ }^{\nexists / / l} \mathrm{Gfap}-\mathrm{Cre}^{+}$mice following intracranial WNV infection.

In light of these findings, we next assessed whether IFN signaling in astrocytes from the cerebellum and cerebral cortex dif- ferentially impacts BBB function. We generated in vitro BBB cultures in which primary murine BMECs derived from the cerebral cortex were cocultured on inserts above astrocytes derived from the cerebellum or cerebral cortex. Measurements of BBB integrity were taken by recording transendothelial electrical resistance (TEER), as described previously (4). While in vitro BBB cultures with cerebral cortex or cerebellar astrocytes did not exhibit baseline differences in TEER, the addition of IFN- $\beta$ to the bottom chamber resulted in an enhancement of TEER in cultures with 
cerebellar astrocytes, beyond that observed in cultures with astrocytes from the cerebral cortex (Figure 7A).

To assess whether astrocytes derived from different brain regions could influence the ability of WNV to cross an intact $\mathrm{BBB}$, we generated in vitro $\mathrm{BBB}$ cultures composed of uniform cultures of WT BMECS grown on inserts above either WT or Ifnar ${ }^{--}$astrocytes derived from either the cerebral cortex or cerebellum. WNV was placed into the top chamber of the cultures for 6 hours, after which viral trafficking was measured by extracting and combining RNA from the supernatants and astrocytes of the bottom chambers. We observed less viral trafficking to the bottom chambers of the cultures with WT cerebellar astrocytes than to those with WT cerebral cortical astrocytes (Figure 7B). However, we did not observe this difference when astrocytes were deficient in IFNAR. Pretreatment of cultures with IFN- $\beta$ effectively limited viral trafficking across in vitro BBB in all cultures, consistent with our previous report showing synergistic effects of IFN signaling in endothelial cells and astrocytes on the modulation of WNV trafficking in vitro (4). However, IFN- $\beta$ treatment suppressed viral trafficking more robustly in cultures with WT cerebellar astrocytes than in those with cerebral cortex astrocytes, and this difference was lost when astrocytes lacked IFNAR expression (Figure 7B), suggesting that cerebellar astrocytes are better able than are cerebral cortical astrocytes to positively regulate $\mathrm{BBB}$ function following stimulation with type I IFN.

Given the differences in the efficacy of IFN signaling in astrocytes derived from different brain regions, we explored whether IFN signaling programs in astrocytes from these regions were different. We compared transcript levels of canonical ISGs between human astrocytes derived from the cerebral cortex and cerebellum. Remarkably, we observed enhanced basal expression of several ISGs, including the PRR TLR7, the transcription factor IFN regulatory factor 3 (IRF3), and other major antiviral or proinflammatory genes (IFN-induced protein with tetratricopeptide repeats 1 [IFIT1], 2'-5'-oligoadenylate synthetase 1 [OAS1], and $\mathrm{C}-\mathrm{X}-\mathrm{C}$ motif chemokine ligand 11 [CXCL11]) (Figure 7, C-H). In contrast, basal expression of other PRR and signaling molecules (e.g., TLR3 and IRF7) did not differ between the 2 types of astrocytes (data not shown). Moreover, astrocytes derived from the cerebellum exhibited enhanced expression of ISGs after 4 hours of treatment with IFN- $\beta$, though by 24 hours, both astrocyte populations reached expression levels equivalent to those of most ISGs. These data suggest that astrocytes from the cerebellum are primed at baseline with enhanced type I IFN signatures compared with astrocytes from the cerebral cortex and reach maximal ISG expression more rapidly following IFN stimulation. This pattern was not unique to human astrocytes. While baseline expression of the type I and type III IFN receptors IFNAR1 and IFNLR1 did not differ, we observed higher basal and IFN-induced expression of ISG mRNAs and proteins in primary murine cerebellar astrocytes compared with expression levels in those from the cerebral cortex (Supplemental Figure 9, A-I).

To generate a more complete picture of differential gene expression, we next performed mRNA sequencing (RNA-seq) in primary human astrocytes from either brain region following 4 hours of treatment with IFN- $\beta$ or a PBS vehicle. Our analysis of global gene expression revealed substantial differential gene expression between astrocytes from cerebral cortex and those from cerebellum across both treatment conditions (Figure 7I). Pathway analysis of these genes revealed that many relevant pathways were differentially regulated in astrocytes from either region, including IFN signaling and IRF activation of cytosolic PRRs (Supplemental Table 1). Of note, other potentially important pathways that exhibited differential regulation included innate immune pathways such as death receptor signaling and retinoic acid-mediated apoptosis; moreover, pathways relevant to BBB biology, including TJ signaling and adherens junction signaling, were also differentially regulated across regions. Further analysis confirmed our previous finding that homeostatic and IFN-induced expression of ISGs and PRRs was higher in cerebellar astrocytes than in astrocytes from cerebral cortex (Supplemental Tables 2 and 3). Examples of IFN-related genes expressed more highly in cerebellar astrocytes include Ifit1, Ifit2, Ifit3, IFN-induced with helicase $\mathrm{C}$ domain 1 (Ifih1, also known as MDA5), IFN- $\gamma$-inducible protein 16 (Ifi16), IFN-induced protein 44 like (Ifi44l), MX dynamin-like GTPase 1 ( $M x 1)$, radical S-adenosyl methionine domain-containing 2 (Rsad2, also known as viperin), and Stat1. While not every gene identified in our quantitative reverse transcription PCR (qRT-PCR) studies met the threshold for statistical significance in our RNA-seq analysis, these studies confirm broad differential regulation of innate immune and IFN signaling pathways in astrocytes isolated from cerebellum compared with those from cerebral cortex.

\section{Discussion}

Alterations in BBB biology and function during neurotropic viral infection have been presumed to arise from uniform responses of BMECs throughout the CNS vasculature. In this study, we demonstrate that innate immune responses of astrocytes from evolutionarily distinct regions of the CNS contribute to BBB heterogeneity via differential activation of antiviral signaling pathways. Our studies indicate differential roles for type I IFN signaling in hindbrain and forebrain astrocytes, as loss of IFNAR signaling in astrocytes resulted in enhanced BBB permeability, early viral neuroinvasion, and increased immunopathologic neuronal cell death in the cerebellum of mice following WNV infection. Studies using both human and mouse astrocytes showed enhanced basal and IFN-induced expression of PRRs and ISGs in cerebellar astrocytes compared with expression levels in cortical astrocytes, suggesting that cerebellar astrocytes are primed for more rapid IFN responses upon detection of CNS pathogens, leading to greater enhancement of BBB function and suppression of neuroinflammation.

These findings increase our understanding of the immunologic heterogeneity of the CNS. A growing body of research has established the differential susceptibility of CNS regions to infection and autoimmunity $(15,18,31,39)$, though the resident CNS cells and signaling pathways that underlie these heterogeneities remain poorly understood. As an example, neurons of hindbrain and forebrain regions exhibit differential type I IFN signaling in response to positive-strand RNA viruses (18), and, in general, type I IFN responses to viruses vary among neuronal subtypes and their stages of differentiation (18, 40-42). While nonspecific neuronal deletion of Ifnar in our study could confound analyses of similar regional phenotypes in astrocytes, the Gfap-Cre line 77.6 has been 

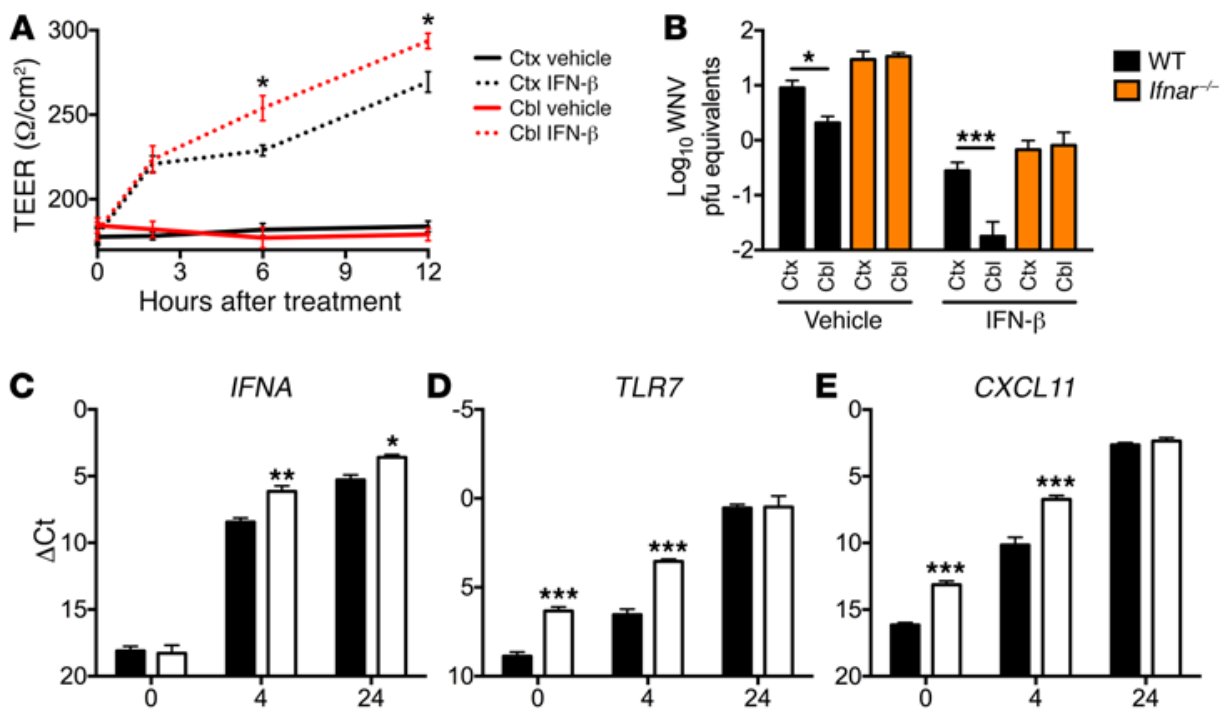

E $\quad$ CXCL11

Hours after treatment

Hours after treatment

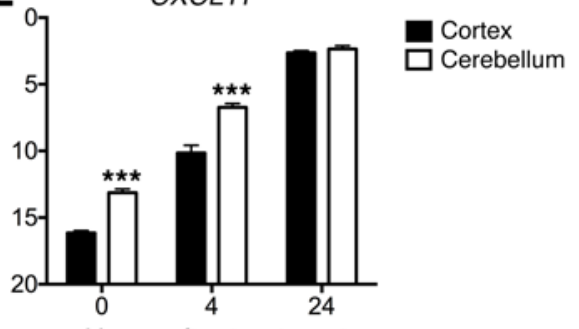

Hours after treatment
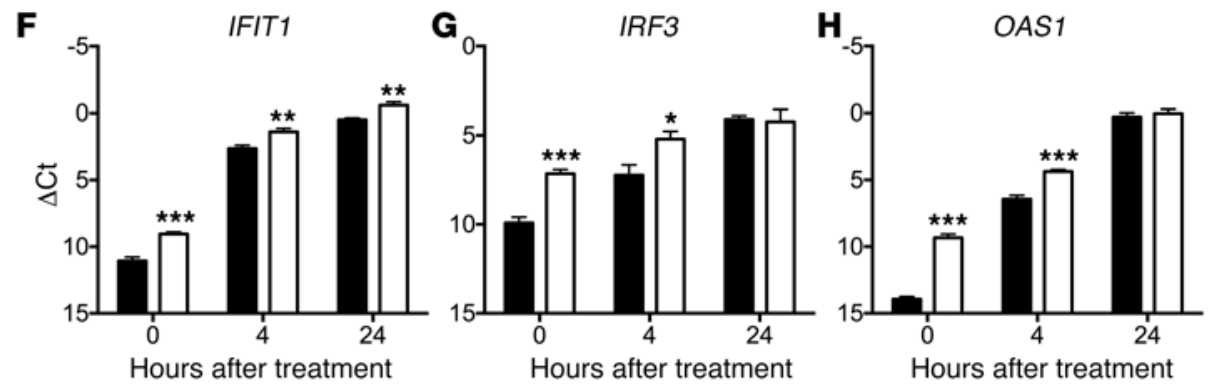

I
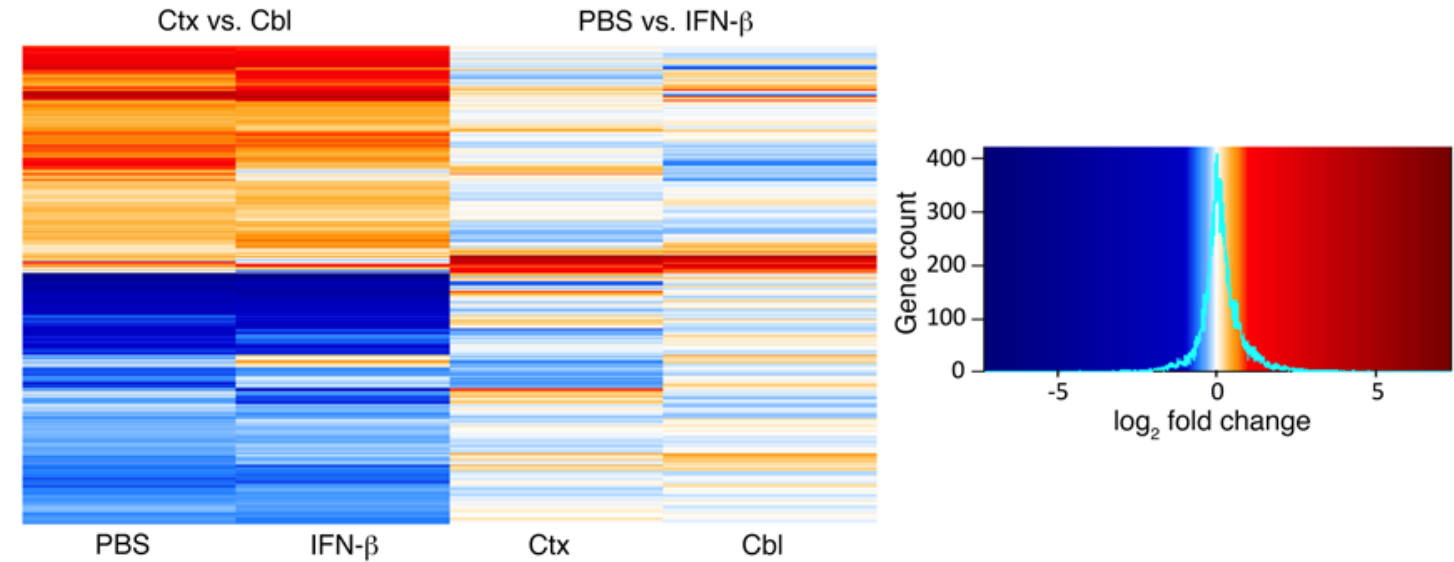

Figure 7. Type I IFN responses in astrocytes in vitro. (A and B) In vitro BBB Transwell cultures were generated with either cerebral cortical or cerebellar astrocytes. (A) TEER recordings in cultures treated with saline vehicle or $10 \mathrm{U} / \mathrm{ml}$ recombinant IFN- $\beta$. (B) Cultures were generated with cerebral cortical or cerebellar astrocytes ( $x$ axis) derived from either WT (black bars) or Ifnar ${ }^{/-}$mice (orange bars) and treated overnight with either saline vehicle or $10 \mathrm{U} / \mathrm{ml}$ recombinant IFN- $\beta$. Following pretreatment, WNV (0.01 MOI) was added to the top chamber of cultures and allowed to migrate for 6 hours. Data represent combined WNV genome copy numbers detected in the astrocyte monolayer and bottom chamber supernatant. (C-H) Primary human cerebral cortical or cerebellar astrocytes were treated with $10 \mathrm{U} / \mathrm{ml}$ recombinant IFN- $\beta$ and analyzed for transcript expression of the indicated genes 4 and 24 hours after treatment. Ct values for all genes were normalized to Ct values of the housekeeping gene GAPDH. (I) Primary human cerebral cortical or cerebellar astrocytes were treated for 4 hours with PBS or $10 \mathrm{U} / \mathrm{ml}$ recombinant IFN- $\beta$ and subjected to RNA-seq. Heatmap and histogram of global gene expression across regions and treatment groups were generated from all statistically significant genes. Data in A-H represent the mean \pm SEM of 5 to 6 replicates from 2 to 3 independent experiments and were analyzed by 2-way ANOVA. Data in I were derived from 3 independent samples per group and were analyzed as described in the Methods. ${ }^{*} P<0.05,{ }^{* *} P<0.01$, and ${ }^{* * *} P<0.001$. Cbl, cerebellum; Ctx, cortex. 
shown in numerous studies to have reliably astrocyte-specific expression (22-25). Consistent with this, we observed deletion of Ifnar on cortical and cerebellar astrocytes, with intact expression of the receptor on nonastrocytic cells. The lack of alterations in virologic and immunologic parameters in the periphery or after intracortical inoculation of virus also suggests that there is little nonspecific deletion of Ifnar in neurons or peripheral cells. Although regional heterogeneity in astrocyte neurophysiologic functions has been appreciated $(15,43,44)$, few studies have evaluated the differential responses of these astrocytes to viral infections. Our study suggests that differential IFN signaling in hindbrain regions is multicellular in nature and may be common to all cells of neuroectodermal lineage in the hindbrain. The confirmation of these findings in neurons and astrocytes from both murine and human sources suggests a phenomenon that may be conserved in recent evolution and that perhaps serves to protect centers of vital autonomic function in the cerebellum and brainstem from pathogenic and immunologic injury.

Our findings highlight the multifaceted contributions of astroglia to the regulation of CNS immune responses. Loss of IFNAR signaling in astrocytes has multiple consequences, including the enhanced expression of inflammatory cytokines and chemokines, which can disrupt the BBB during neurotropic viral infection $(4,6$, 7). This enhanced $\mathrm{BBB}$ permeability is associated with earlier detection of WNV in hindbrain regions of the CNS, suggesting that astrocyte IFNAR signaling prevents or delays infection at this site. Altered expression of cytokines such as IL-1 $\beta$ also probably influences other functions of the BBB, including local antigen presentation $(21,45)$ and perivascular capture of infiltrating leukocytes $(9,46)$, as reflected by the broad parenchymal localization of $\mathrm{CD} 3^{+}$lymphocytes in our study. Regional differences in astrocyte signaling may also differentially impact regional differences in other neurovascular cell types, including endothelial cells. While WNV may enter the CNS via multiple mechanisms, including nonhematogenous routes such as retrograde axonal transport (47) and transynaptic trafficking (48), the critical role of BBB function in limiting WNV neuroinvasion and subsequent disease burden has been established in many recent studies $(4,27,28)$. Moreover, sciatic nerve ligation ipsilateral to the footpad used for s.c. inoculation does not rescue CNS infection or mortality in mice (47), suggesting that hematogenous spread alone is sufficient for severe neuroinvasive infection. Thus, the alterations to BBB function observed in our study probably contributed significantly to the enhanced disease pathogenesis we observed in Ifnar ${ }^{\text {t/f }}$ Gfap-Cre ${ }^{+}$mice following WNV infection.

IFNAR signaling in astrocytes was important for the suppression of VCAM-1 expression in neurovascular cells of the hindbrain. A potential mechanism for this effect is type I IFN-mediated suppression of inflammatory cytokines. For example, several recent studies have described a role for type I IFN in suppressing the expression of IL-1 $(4,49,50)$, which is known to induce VCAM-1 expression in astrocytes (51,52). VCAM-1-VLA-4 interactions facilitate immune trafficking during CNS infection $(38,53)$. Although CNS immune infiltration is required for viral clearance following WNV infection $(9,54)$, high levels of inflammatory cell infiltration are associated with pathology and bystander injury (11, $45,55)$. Thus, astrocyte IFNAR-mediated suppression of VCAM-1 expression appears to play a protective antiinflammatory role in hindbrain tissues. This role is supported by studies demonstrating that pharmacological blockade of VLA-4 in the context of neurotropic viral infection can ameliorate disease, largely via suppression of CNS immunopathology $(38,53)$. We note, however, that the experimental consequences of VLA-4 blockade are likely context dependent, varying by disease model and timing of treatment after infection, and that this blockade may act through additional VLA-4 ligands beyond VCAM-1, including JAM-B and fibronectin (56). Moreover, modulation of VCAM-1 is likely only one of several relevant regulatory outputs of type I IFN signaling in astrocytes and probably acts in concert with independent effects on inflammatory cytokine expression and BBB function to coordinate neuroinflammation during WNV infection.

Our study demonstrating a role for astrocytes in limiting inflammation via the regulation of BBB permeability has clinical implications. Loss of BBB integrity in the hindbrain, as evidenced by gadolinium contrast-enhanced magnetic resonance imaging (Gd-MRI), is associated with poor prognosis in patients with WNV neuroinvasive disease $(19,57)$. As Gd-MRI-enhanced lesions develop in conjunction with inflammatory infiltrates, alteration in vascular permeability is believed to occur in tandem with the capture and trafficking of leukocytes from the blood at endothelial barriers $(58,59)$. Indeed, recent studies indicate that increased BBB permeability predicts the development of neuroinflammation in patients with CNS autoimmunity (60). Although impaired VLA-4 function is known to jeopardize host protection during acute viral infection of the CNS (61), our study suggests that patients with acute WNV encephalitis and persistent cerebellar inflammation and disease symptoms might benefit from VLA-4 blockade. In summary, these findings advance our understanding of astrocyte innate immune responses during viral infection, identify regionally distinct roles for astrocytes in the regulation of BBB function, and suggest that defining signaling pathways that modulate leukocyte entry into distinct brain regions can identify new targets for the prevention and treatment of CNS immunopathology.

\section{Methods}

Mouse models of WNV encephalitis. All animals used in this study were infected at 8 weeks of age. The Gfap-Cre ${ }^{+}$line 77.6 was obtained commercially from The Jackson Laboratory and maintained in the hemizygous state. Ifnar ${ }^{f / f l}$ mice were obtained from R. Schreiber (Washington University School of Medicine, St. Louis, Missouri, USA) and U. Kalinke (Paul-Ehrlich-Institut, Hannover, Germany). Ifnarl/fll mice (62) were backcrossed using speed congenic analysis to $99 \%$ C57BL/6J as judged by microsatellite analysis. All animals were housed under specific pathogen-free conditions. Littermates for the survival studies performed and depicted in Figure 1A were derived from crosses of Ifnar ${ }^{f / W T}$ Gfap-Cre ${ }^{+}$with Ifnar ${ }^{f / / f l}$ breeders. Subsequently, littermates were obtained from breeders on a homozygous Ifnar ${ }^{f / / l}$ background (Ifnar $^{f / f l} \mathrm{Gfap}-\mathrm{Cre}^{+/-}$with Ifnar $\left.{ }^{f / f t}\right)$. Inoculation via footpad s.c. injection $(50 \mu \mathrm{l})$ or intracranial inoculation $(10 \mu \mathrm{l})$ was performed with either $10^{2}$ or $10 \mathrm{PFU}$ of WNV, respectively, as previously described (63). WNV (strain 3000.0259, isolated in New York in 2000) (64) was used in all experiments except those depicted in Supplemental Figure 5 , in which the WNV-Madagascar (strain DakAnMg798, isolated in 1978) (65) also was used. Viral titers in all studies were assessed via plaque assay in BHK21-15 cells, as previously described (66). 
Immunohistochemical analysis. CNS tissue sections were obtained after cardiac perfusion with $4 \%$ paraformaldehyde in PBS. Tissues were frozen, blocked, and stained as previously described (31) with primary antibodies against IFNAR1 (BioLegend; 127305); S100- $\beta$ (Abcam; ab41548); CD31/endomucin antibody cocktail (BD, 550274 and eBioscience, 14-5851-81) (67); VCAM-1 (Abcam; ab134047); ALDH1L1 (Abcam; ab52492); NeuN (EMD Millipore; MAB277B); and CD3 (Abcam; ab16044). Secondary fluorescence staining was performed with the appropriate Alexa Fluor 488 and 555 antibodies (Life Technologies, Thermo Fisher Scientific). Nuclei in all preparations were stained with TO-PRO-3 (Life Technologies, Thermo Fisher Scientific). TUNEL staining was performed with a TMR Red In Situ Cell Death Detection Kit (Roche). All images were acquired via confocal microscopy (Carl Zeiss). Image analysis was performed with Image J software (NIH). Details on individual analyses are provided in the figure legends.

In vivo assessment of $B B B$ permeability. Sodium fluorescein permeability experiments were performed as previously described (4). Briefly, on predetermined days following infection, mice were injected i.p. with $100 \mu \mathrm{l}$ of $100 \mathrm{mg} / \mathrm{ml}$ fluorescein sodium salt (SigmaAldrich) in sterile PBS. Tissue homogenates and serum were collected following perfusion with PBS and then incubated overnight in $2 \%$ TCA (Sigma-Aldrich) to precipitate protein. Supernatants were diluted in equal volumes of borate buffer, $\mathrm{pH} 11.0$ (Sigma-Aldrich). Tissue fluorescence values were determined with a fluorometric plate reader and standardized against plasma fluorescence values for individual mice. Fluorescence staining for extravasated endogenous IgG was performed as previously described (27).

CNS leukocyte isolation and flow cytometric analysis. Leukocytes were isolated from freshly dissected CNS regions following PBS perfusion and stained with fluorescently conjugated antibodies to CD3, CD4, CD8 $\beta$, CD11b, F4/80, and CD45 as previously described (21). Intracellular IFN- $\gamma$ staining was performed on isolated leukocytes in an I-A ${ }^{b}$-restricted NS3-2066 and NS3-1616 peptide and a $\mathrm{D}^{\mathrm{b}}$-restricted NS4B peptide restimulation assay as previously described (27). An LSR Flow Cytometer (BD) and FlowJo software (Tree Star) were used for data acquisition and analysis.

qRT-PCR analysis. Total RNA was collected from freshly dissected CNS regions following PBS perfusion or from cell cultures using an RNeasy Kit (QIAGEN) according to the manufacturer's instructions. Reverse transcription and SYBR Green qRT-PCR were performed for cytokine, chemokine, and ISG analytes as previously described (31). Ct values for all genes were normalized to $\mathrm{Ct}$ values of the housekeeping gene GAPDH. Data from qRT-PCR experiments are reported as $\triangle \mathrm{Ct}$ $\left(\mathrm{Ct}_{\text {target }}-\mathrm{Ct}_{\mathrm{GAPDH}}\right)$. Primers used for all analyses are reported in Supplemental Table 4. qRT-PCR analysis of WNV copy numbers in serum and cell culture supernatants was performed with TaqMan reagents (Thermo Fisher Scientific) as previously described $(27,66)$.

Western blotting. Protein lysates were collected in radioimmunoprecipitation assay (RIPA) buffer (Sigma-Aldrich), separated on NuPage 4\%-12\% Bis-Tris Gels (Invitrogen, Thermo Fisher Scientific), and transferred to nitrocellulose membranes via the iBlot 2 transfer system (Thermo Fisher Scientific). Membranes were incubated overnight at $4^{\circ} \mathrm{C}$ in TBS plus $4 \%$ powdered milk with the following primary antibodies: anti-IFIT1 mAb (13B4-1, unpublished antibody developed in the laboratory of Michael Diamond); anti-IFIT2 polyclonal antibody (Thermo Fisher Scientific; PA3-845); and anti- $\beta$ actin mAb (Cell Sig- naling Technology; 93700P). Membranes were washed 3 times with water, followed by a 3-hour incubation at room temperature with anti-mouse DyLight 800 (Thermo Fisher Scientific; 52575) and goat anti-rabbit IRDye 680RD (LI-COR; 925-68071) secondary antibodies. Membranes were washed 3 times with TBS Tween-20 (TBST) and imaged using the Odyssey Gel Imaging System (LI-COR).

Astrocyte and in vitro BBB cultures. Primary cerebral cortex and cerebellar human astrocytes were obtained from ScienCell Research Laboratories (catalogs 1800 and 1810) and grown according to the manufacturer's protocols in ScienCell Astrocyte Medium (catalog 1801). Primary neonatal mouse astrocytes were obtained and maintained as previously described (4). Immunohistochemical characterization of primary human and murine astrocytes using anti-S100- $\beta$ (Abcam; ab41548) and anti-ALDH1A1 (Abcam; ab52492) antibodies in conjunction with nuclear stains (TO-PRO-3) revealed a greater than $99 \%$ purity. In vitro BBB cultures were generated with primary mouse BMECs and astrocytes and used in TEER and viral migration assays as previously described $(4,27)$.

IFN treatment. Astrocyte and in vitro BBB cultures were treated in normal culture medium with $10 \mathrm{U} / \mathrm{ml}$ mouse or human recombinant IFN- $\beta$ (PBL Assay Sciences) or 0.1\% BSA in PBS vehicle, as appropriate. Cytokines were added to the bottom chamber of in vitro BBB cultures. Endothelial cells and astrocytes remained in coculture for the duration of the cytokine treatment.

VLA-4 blockade studies. The VLA-4 antagonist BIO5192 (Tocris Bioscience) was reconstituted in aqueous vehicle containing ethanol and propylene glycol as described previously (56). A daily dosage of 10 $\mathrm{mg} / \mathrm{kg}$ was chosen on the basis of published studies of serum half-life and receptor occupancy $(56,68)$. All injections were given i.p. beginning on day 4 after WNV infection.

RNA-seq. Human astrocytes from cerebral cortex or cerebellum were cultured and treated for 4 hours with PBS or recombinant IFN- $\beta$ as described above. Total RNA was extracted using an RNeasy Kit (QIAGEN) and prepared for sequencing. Raw RNA-seq data (FASTQ files) were demultiplexed and checked for quality (FASTQC), and adapters and rRNA were digitally removed (cutadapt, version 1.8.3 and Bowtie2, version 2.2.5). Sufficient reads were generated ( $\sim 30$ million reads) and then mapped to the human genome (NCBI build 37.1). Raw reads were aligned to the host genome using STAR (2.4.2) and then converted into gene counts with HTSeq (version 0.6.0). Gene counts were filtered by a mean of 10 or greater across all samples. Exploratory analysis and statistics were run using the $\mathrm{R}$ statistical programming language (version 3.2.0). The gene count matrix was normalized using voom through the Bioconductor limma package (version 3.26.9). Statistical analysis (including differential expression) was performed using $\mathrm{R}$ and Bioconductor. Sequencing data were deposited in the NCBI's Gene Expression Omnibus (GEO) database (GEO GSE89476).

Statistics. Survival experiments were analyzed by log-rank test. Viral titers in tissue were analyzed by Mann-Whitney $U$ test. Other experiments were analyzed with parametric tests (2-tailed Student's $t$ test or 2-way ANOVA), with correction for multiple comparisons where appropriate. All statistical analysis except for RNA-seq analysis was performed using GraphPad Prism Version 7 software (GraphPad Software). A $P$ value of less than 0.05 was considered statistically significant.

Study approval. All experiments were performed in compliance with and under the approval of the Washington University Animal Studies Committee. 


\section{Author contributions}

Conceptualization: BPD and RSK; methodology: BPD, MSD, and RSK; analysis: BPD, HJ, DMD, JLW, RRG, and HML; investigation: BPD, HJ, DMD, JLW, JPW, and HML; writing of the original draft: BPD and RSK; review and editing of the manuscript: BPD, JLW, HML, MSD, and RSK; supervision and funding acquisition: MG, MSD, and RSK.

\section{Acknowledgments}

We thank Denise Dorsey, Qingping Wu, and Matthew Cain for their technical assistance (Washington University School of Medicine, St. Louis, MO, USA). We also thank the University of Washington's Center for Innate Immunity and Immune Disease Immuno-Informatics core group for their bioinformatics support. This work was supported by NIH grants U19 AI083019 (to RSK, MSD, and MG), R01 NS052632 (to RSK), and R01 AI104002 (to MG). BPD was supported by a National Science Foundation Graduate Research Fellowship (DGE-1143954) and an NIH NRSA fellowship (F31NS07866). JLW was supported by a postdoctoral fellowship from the National Multiple Sclerosis Society. JPW was supported by an NIH NRSA fellowship (F32-AI112274). Experimental support was provided by the Speed Congenics Facility of the Rheumatic Diseases Core Center (Washington University School of Medicine, St. Louis, MO, USA). Research reported in this article was supported by the National Institute of Arthritis and Musculoskeletal and Skin Diseases, part of the NIH, under award number P30AR048335. The content is solely the responsibility of the authors and does not necessarily represent the official views of the NIH.

Address correspondence to: Robyn S. Klein, 660 S. Euclid Avenue, St. Louis, Missouri 63110, USA. Phone: 314.286.2140; E-mail: rklein@wustl.edu.
1. Platanias LC. Mechanisms of type-I- and type-II-interferon-mediated signalling. Nat Rev Immunol. 2005;5(5):375-386.

2. Lazear HM, Diamond MS. New insights into innate immune restriction of West Nile virus infection. Curr Opin Virol. 2015;11:1-6.

3. Schoggins JW, Rice CM. Interferon-stimulated genes and their antiviral effector functions. Curr Opin Virol. 2011;1(6):519-525.

4. Daniels BP, Holman DW, Cruz-Orengo L, Jujjavarapu H, Durrant DM, Klein RS. Viral pathogen-associated molecular patterns regulate blood-brain barrier integrity via competing innate cytokine signals. MBio. 2014;5(5):e01476-e01414.

5. Persidsky Y, Ramirez SH, Haorah J, Kanmogne GD. Blood-brain barrier: structural components and function under physiologic and pathologic conditions. J Neuroimmune Pharmacol. 2006;1(3):223-236.

6. Daniels BP, Klein RS. Viral sensing at the bloodbrain barrier: new roles for innate immunity at the CNS vasculature. Clin Pharmacol Ther. 2015;97(4):372-379.

7. Pan W, Stone KP, Hsuchou H, Manda VK, Zhang Y, Kastin AJ. Cytokine signaling modulates blood-brain barrier function. Curr Pharm Des. 2011;17(33):3729-3740.

8. Chai Q, He WQ, Zhou M, Lu H, Fu ZF. Enhancement of blood-brain barrier permeability and reduction of tight junction protein expression are modulated by chemokines/cytokines induced by rabies virus infection. J Virol. 2014;88(9):4698-4710.

9. McCandless EE, Zhang B, Diamond MS, Klein RS. CXCR4 antagonism increases T cell trafficking in the central nervous system and improves survival from West Nile virus encephalitis. Proc Natl Acad Sci USA. 2008;105(32):11270-11275.

10. Zhao L, Toriumi H, Kuang Y, Chen H, Fu ZF. The roles of chemokines in rabies virus infection: overexpression may not always be beneficial. J Virol. 2009;83(22):11808-11818.

11. Wang Y, Lobigs M, Lee E, Müllbacher A. CD8+ $\mathrm{T}$ cells mediate recovery and immunopathology in West Nile virus encephalitis. J Virol. 2003;77(24):13323-13334.

12. Furr SR, Marriott I. Viral CNS infections: role of glial pattern recognition receptors in neuroinflammation. Front Microbiol. 2012;3:201.

13. Furr SR, Moerdyk-Schauwecker M, Grdzelishvili VZ, Marriott I. RIG-I mediates nonsegmented negative-sense RNA virus-induced inflammatory immune responses of primary human astrocytes. Glia. 2010;58(13):1620-1629.

14. Pfefferkorn C, et al. Abortively infected astrocytes appear to represent the main source of interferon beta in the virus-infected brain. JVirol. 2015;90(4):2031-2038.

15. Zhang Y, Barres BA. Astrocyte heterogeneity: an underappreciated topic in neurobiology. Curr Opin Neurobiol. 2010;20(5):588-594.

16. Morga E, Faber C, Heuschling P. Regional heterogeneity of the astroglial immunoreactive phenotype: effect of lipopolysaccharide. J Neurosci Res. 1999;57(6):941-952.

17. Fitting S, Zou S, Chen W, Vo P, Hauser KF, Knapp $\mathrm{PE}$. Regional heterogeneity and diversity in cytokine and chemokine production by astroglia: differential responses to HIV-1 Tat, gp120, and morphine revealed by multiplex analysis. JProteome Res. 2010;9(4):1795-1804.

18. Cho H, Proll SC, Szretter KJ, Katze MG, Gale M, Diamond MS. Differential innate immune response programs in neuronal subtypes determine susceptibility to infection in the brain by positive-stranded RNA viruses. Nat Med. 2013;19(4):458-464.

19. Hart J, et al. West Nile virus neuroinvasive disease: neurological manifestations and prospective longitudinal outcomes. BMC Infect Dis. 2014;14:248.

20. Shrestha B, Gottlieb D, Diamond MS. Infection and injury of neurons by West Nile encephalitis virus. J Virol. 2003;77(24):13203-13213.

21. Durrant DM, Daniels BP, Klein RS. IL-1R1 signaling regulates CXCL12-mediated T cell localization and fate within the central nervous system during West Nile Virus encephalitis. JImmunol. 2014;193(8):4095-4106.

22. Tao J, et al. Deletion of astroglial Dicer causes non-cell-autonomous neuronal dysfunction and degeneration. J Neurosci. 2011;31(22):8306-8319.

23. Niu W, et al. In vivo reprogramming of astrocytes to neuroblasts in the adult brain. Nat Cell Biol. 2013;15(10):1164-1175.
24. Howng SY, Huang Y, Ptáček L, Fu YH. Understanding the role of dicer in astrocyte development. PLoS ONE. 2015;10(5):e0126667.

25. Molumby MJ, Keeler AB, Weiner JA. Homophilic protocadherin cell-cell interactions promote dendrite complexity. Cell Rep. 2016;15(5):1037-1050.

26. Pinto AK, et al. Deficient IFN signaling by myeloid cells leads to MAVS-dependent virus-induced sepsis. PLoS Pathog. 2014;10(4):e1004086.

27. Lazear HM, et al. Interferon- $\lambda$ restricts West Nile virus neuroinvasion by tightening the blood-brain barrier. Sci Transl Med. 2015;7(284):284ra59.

28. Miner JJ, et al. The TAM receptor Mertk protects against neuroinvasive viral infection by maintaining blood-brain barrier integrity. Nat Med. 2015;21(12):1464-1472.

29. Tedeschi B, Barrett JN, Keane RW. Astrocytes produce interferon that enhances the expression of $\mathrm{H}-2$ antigens on a subpopulation of brain cells. JCell Biol. 1986;102(6):2244-2253.

30. Keller BC, et al. Resistance to alpha/beta interferon is a determinant of West Nile virus replication fitness and virulence. J Virol. 2006;80(19):9424-9434.

31. Klein RS, et al. Neuronal CXCL10 directs CD8+ T-cell recruitment and control of West Nile virus encephalitis. J Virol. 2005;79(17):11457-11466.

32. Floris $\mathrm{S}$, et al. Interferon-beta directly influences monocyte infiltration into the central nervous system. J Neuroimmunol. 2002;127(1-2):69-79.

33. Bitsch $\mathrm{A}$, et al. Interferon beta-1b modulates serum sVCAM-1 levels in primary progressive multiple sclerosis. Acta Neurol Scand. 2004;110(6):386-392.

34. Calabresi PA, et al. Increases in soluble VCAM-1 correlate with a decrease in MRI lesions in multiple sclerosis treated with interferon beta-1b. Ann Neurol. 1997;41(5):669-674.

35. Engelhardt B, Ransohoff RM. Capture, crawl, cross: the T cell code to breach the blood-brain barriers. Trends Immunol. 2012;33(12):579-589.

36. Gimenez MA, Sim JE, Russell JH. TNFR1-dependent VCAM-1 expression by astrocytes exposes the CNS to destructive inflammation. J Neuroimmunol. 2004;151(1-2):116-125. 
37. Archambault AS, Sim J, McCandless EE, Klein RS, Russell JH. Region-specific regulation of inflammation and pathogenesis in experimental autoimmune encephalomyelitis. J Neuroimmunol. 2006;181(1-2):122-132.

38. Getts DR, et al. Targeted blockade in lethal West Nile virus encephalitis indicates a crucial role for very late antigen (VLA)-4-dependent recruitment of nitric oxide-producing macrophages. J Neuroinflammation. 2012;9:246.

39. Stromnes IM, Cerretti LM, Liggitt D, Harris RA, Goverman JM. Differential regulation of central nervous system autoimmunity by $\mathrm{T}(\mathrm{H}) 1$ and $\mathrm{T}(\mathrm{H}) 17$ cells. Nat Med. 2008;14(3):337-342.

40. Rosato PC, Leib DA. Neuronal interferon signaling is required for protection against herpes simplex virus replication and pathogenesis. PLOS Pathog. 2015;11(7):e1005028.

41. Farmer JR, Altschaefl KM, O'Shea KS, Miller DJ. Activation of the type I interferon pathway is enhanced in response to human neuronal differentiation. PLoS ONE. 2013;8(3):e58813.

42. Schultz KL, Vernon PS, Griffin DE. Differentiation of neurons restricts Arbovirus replication and increases expression of the alpha isoform of IRF-7. J Virol. 2015;89(1):48-60.

43. Molofsky AV, et al. Astrocyte-encoded positional cues maintain sensorimotor circuit integrity. Nature. 2014;509(7499):189-194.

44. Sosunov AA, Wu X, Tsankova NM, Guilfoyle E, McKhann GM, Goldman JE. Phenotypic heterogeneity and plasticity of isocortical and hippocampal astrocytes in the human brain. J Neurosci. 2014;34(6):2285-2298.

45. Durrant DM, Robinette ML, Klein RS. IL-1R1 is required for dendritic cell-mediated $\mathrm{T}$ cell reactivation within the CNS during West Nile virus encephalitis. JExp Med. 2013;210(3):503-516.

46. McCandless EE, Budde M, Lees JR, Dorsey D, Lyng E, Klein RS. IL-1R signaling within the central nervous system regulates CXCL12 expression at the blood-brain barrier and disease severity during experimental autoimmune encephalomyelitis. JImmunol. 2009;183(1):613-620.
47. Samuel MA, Wang H, Siddharthan V, Morrey JD, Diamond MS. Axonal transport mediates West Nile virus entry into the central nervous system and induces acute flaccid paralysis. Proc Natl Acad Sci USA. 2007;104(43):17140-17145.

48. Maximova OA, Bernbaum JG, Pletnev AG. West Nile virus spreads transsynaptically within the pathways of motor control: Anatomical and ultrastructural mapping of neuronal virus infection in the primate central nervous system. PLoS Negl Trop Dis. 2016;10(9):e0004980.

49. Guarda G, et al. Type I interferon inhibits interleukin-1 production and inflammasome activation. Immunity. 2011;34(2):213-223.

50. Inoue $M$, Shinohara ML. The role of interferon- $\beta$ in the treatment of multiple sclerosis and experimental autoimmune encephalomyelitis - in the perspective of inflammasomes. Immunology. 2013;139(1):11-18.

51. Moynagh PN. The interleukin-1 signalling pathway in astrocytes: a key contributor to inflammation in the brain. J Anat. 2005;207(3):265-269.

52. An Y, Chen Q, Quan N. Interleukin-1 exerts distinct actions on different cell types of the brain in vitro. J Inflamm Res. 2011;2011(4):11-20.

53. Campbell JH, et al. Anti- $\alpha 4$ antibody treatment blocks virus traffic to the brain and gut early, and stabilizes CNS injury late in infection. PLoS Pathog. 2014;10(12):e1004533.

54. Shrestha B, Diamond MS. Role of CD8+ T cells in control of West Nile virus infection. J Virol. 2004;78(15):8312-8321.

55. Szretter KJ, et al. 2'-O methylation of the viral mRNA cap by West Nile virus evades ifit1-dependent and -independent mechanisms of host restriction in vivo. PLoS Pathog. 2012;8(5):e1002698.

56. Ramirez P, et al. BIO5192, a small molecule inhibitor of VLA-4, mobilizes hematopoietic stem and progenitor cells. Blood. 2009;114(7):1340-1343.

57. Ali M, Safriel Y, Sohi J, Llave A, Weathers S. West Nile virus infection: MR imaging findings in the nervous system. AJNR Am J Neuroradiol.
2005;26(2):289-297.

58. Wang HY, Matsui M, Saida T. Immunological disturbances in the central nervous system linked to MRI findings in multiple sclerosis. J Neuroimmunol. 2002;125(1-2):149-154.

59. Khoury SJ, Guttmann CR, Orav EJ, Kikinis R, Jolesz FA, Weiner HL. Changes in activated T cells in the blood correlate with disease activity in multiple sclerosis. Arch Neurol. 2000;57(8):1183-1189.

60. Cramer SP, Modvig S, Simonsen HJ, Frederiksen JL, Larsson HB. Permeability of the blood-brain barrier predicts conversion from optic neuritis to multiple sclerosis. Brain. 2015;138(Pt 9):2571-2583.

61. Rothhammer V, et al. $\alpha 4$-integrins control viral meningoencephalitis through differential recruitment of T helper cell subsets. Acta Neuropathol Commun. 2014;2:27.

62. Le Bon A, et al. Direct stimulation of T cells by type I IFN enhances the CD8+ T cell response during cross-priming. JImmunol. 2006;176(8):4682-4689.

63. Engle MJ, Diamond MS. Antibody prophylaxis and therapy against West Nile virus infection in wild-type and immunodeficient mice. J Virol. 2003;77(24):12941-12949.

64. Ebel GD, et al. Partial genetic characterization of West Nile virus strains, New York State, 2000. Emerging Infect Dis. 2001;7(4):650-653.

65. Beasley DW, Li L, Suderman MT, Barrett AD. Mouse neuroinvasive phenotype of West Nile virus strains varies depending upon virus genotype. Virology. 2002;296(1):17-23.

66. Brien JD, Lazear HM, Diamond MS. Propagation, quantification, detection, and storage of West Nile virus. Curr Protoc Microbiol. 2013;31:15D.3.1-15D.3.18.

67. Lu ZH, et al. The myeloid-binding peptide adenoviral vector enables multi-organ vascular endothelial gene targeting. Lab Invest. 2014;94(8):881-892.

68. Leone DR, et al. An assessment of the mechanistic differences between two integrin alpha 4 beta 1 inhibitors, the monoclonal antibody TA-2 and the small molecule BIO5192, in rat experimental autoimmune encephalomyelitis. J Pharmacol Exp Ther. 2003;305(3):1150-1162. 